\title{
Addendum zu den S3-Leitlinien Morbus Crohn und Colitis ulcerosa: Betreuung von Patienten mit chronisch entzündlichen Darmerkrankungen in der COVID-19-Pandemie - offene Fragen und Antworten
}

\section{Addendum to S3-Guidelines Crohn's disease and ulcerative colitis: Management of Patients with Inflammatory Bowel Disease in the COVID-19 Pandemic - open questions and answers}

Autoren

Andreas Stallmach ${ }^{1 *}$, Andreas Sturm ${ }^{2 *}$, Irina Blumenstein ${ }^{3}$, Ulf Helwig ${ }^{4}$, Sibylle Koletzko ${ }^{5}$, Petra Lynen $^{6}$, Carsten Schmidt ${ }^{7}$, Axel Dignaß ${ }^{8 *}$, Torsten Kucharzik ${ }^{*}$

Collaborators:

K. Aden, T. Andus, R. Atreya, F. Autschbach, O. Bachmann, D. Bettenworth, U. Böcker, B. Bokemeyer, T. Brechmann,

P. Bufler, J. Büning, J. Däbritz, J. de Laffolie, R. Ehehalt, Ph. Esters, K. Fellermann, St. Fichtner-Feigl, M. Götz, C. Gross,

F. Hartmann, P. Hartmann, W. Häuser, K. Herrlinger, J. C. Hoffmann, S. In der Smitten, B. Kaltz, L. Kanbach,

K. Kannengießer, K.-M. Keller, P. Kienle, J. Klaus, M. Kreis, A.-J. Kroesen, W. Kruis, T. Kühbacher, J. Langhorst, L. Leifeld,

A. Lügering, Ch. Maaser, H. Matthes, G. Moog, J. H. Niess, J. Ockenga, C. Ott, A. Pace, J. Preiß, M. Reinshagen, E. Rijcken,

E. Schnoy, A. Schreyer, B. Siegmund, E. F. Stange, J. Stein, N. Teich, A. Thomann, Ch. Treese, C. Veltkamp, J. Walldorf,

S. Zeißig, J. Zemke

Institute

1 Klinik für Innere Medizin IV (Gastroenterologie, Infektiologie und Hepatologie), Universitätsklinikum Jena, Jena

2 Klinik für Innere Medizin, Schwerpunkt Gastroenterologie, DRK Kliniken Berlin | Westend, Berlin

3 Medizinische Klinik 1 (Gastroenterologie und Hepatologie, Pneumologie und Allergologie, Endokrinologie und Diabetologie sowie Ernährungsmedizin), Universitätsklinikum Frankfurt, Goethe-Universität, Frankfurt

4 Internistische Praxengemeinschaft Oldenburg, Oldenburg

5 Kinderklinik und Kinderpoliklinik im Dr. von Hauner Kinderspital, LMU Klinikum der Universität München, München

6 Deutsche Gesellschaft für Gastroenterologie, Verdauungsund Stoffwechselerkrankungen, Berlin

7 Medizinische Klinik II (Gastroenterologie, Hepatologie, Endokrinologie, Diabetologie und Infektiologie), Klinikum Fulda, Universitätsmedizin Marburg - Campus Fulda, Fulda

8 Medizinische Klinik I, Agaplesion Markus Krankenhaus, Frankfurt

9 Klinik für Innere Medizin Gastroenterologie, Klinikum Lüneburg, Lüneburg
Die Erstautoren AS und AS sowie die Letztautoren AD und TK fungieren als gleichberechtige Autoren.
Bibliografie

DOI https://doi.org/10.1055/a-1193-5475

Z Gastroenterol 2020; 58: 672-692

(c) Georg Thieme Verlag KG, Stuttgart · New York

ISSN 0044-2771

Korrespondenzadresse

Andreas Stallmach

Klinik für Innere Medizin IV

Universitätsklinikum Jena, Am Klinikum 1, 07743 Jena

Tel.: ++ 49/3641/9324401

Fax: $++49 / 3641 / 9324222$

Andreas.stallmach@med.uni-jena.de

$\circledast$ Supplemental Material:

https://doi.org/10.1055/a-1193-5475

\section{ZUSAMMENFASSUNG}

Die COVID-19-Pandemie ist ein weltweiter Ausbruch von neu aufgetretenen Infektionen mit dem SARS-CoV-2-Virus, von denen weltweit derzeit mehr als 10.670.000 Menschen erkrankt sind bzw. waren. In Deutschland leiden ca. 450.000 Patienten an einer chronisch entzündlichen Darmerkrankung; diese Patienten benötigen in der Regel eine kontinuierliche und kompetente Betreuung. Vor dem Hintergrund eines rasch zunehmenden Wissenszuwachses haben 68 Experten, die die derzeit gültigen Leitlinien der DGVS zum Morbus Crohn und zur Colitis ulcerosa erstellt haben, im Rahmen einer virtuellen Konferenz aktuelle und praxisnahe Empfehlungen formuliert, 
um die Versorgung von CED-Patienten zu verbessern. Diese adressieren das Infektionsrisiko einschließlich des Risikos für besondere Gruppen, den möglichen Verlauf der Erkrankung und die Konsequenzen für die medikamentöse und die opera- tive Therapie der Grunderkrankung sowie allgemeine Maßnahmen zur Infektionsprävention und adjuvante Präventionsund Therapiemöglichkeiten.

\section{Herausgeber}

\section{Federführende Fachgesellschaft}

Deutsche Gesellschaft für Gastroenterologie, Verdauungs- und Stoffwechselkrankrankheiten (DGVS)

T. Brechmann (Bochum), A. Stallmach (Jena), B. Bokemeyer (Minden), K. Aden (Kiel), T. Andus (Stuttgart), R. Atreya (Erlangen), O. Bachmann (Pforzheim), D. Bettenworth (Münster), I. Blumenstein (Frankfurt am Main), U. Böcker (Berlin), J. Büning (Lübeck), A. Dignaß (Frankfurt), R. Ehehalt (Heidelberg), P. Esters (Frankfurt), K. Fellermann (Freudenstadt), M. Götz (Böblingen), W. Häuser (Saarbrücken), F. Hartmann (Frankfurt), U. Helwig (Oldenburg), K. Herrlinger (Hamburg), J. Hoffmann (Ludwigshafen), K. Kannengießer (Lüneburg), J. Klaus (Ulm), W. Kruis (Freimersdorf/Pulheim), T. Kucharzik (Lüneburg), T. Kühbacher (Nürtingen), J. Langhorst (Bamberg), A. Lügering (Münster), C. Maaser (Lüneburg), H. Matthes (Berlin), G. Moog (Kassel), J. Niess (Basel), C. Ott (Regensburg), A. Pace (Neumünster), J. Preiß (Berlin), M. Reinshagen (Braunschweig), C. Schmidt (Fulda), B. Siegmund (Berlin), E. Stange (Stuttgart), J. Stein (Frankfurt), A. Sturm (Berlin), N. Teich (Leipzig), A. Thomann (Mannheim), C. Treese (Berlin), C. Veltkamp (Heidelberg), J. Walldorf (Halle), S. Zeißig (Dresden)

\section{Repräsentativität der Leitliniengruppe/Beteiligte Fachgesellschaften}

- DGP (Deutsche Gesellschaft für Pathologie)/BDP (Bundesverband Deutscher Pathologen)

F. Autschbach (Heilbronn)

- DCCV e. V. (Deutsche Morbus Crohn/Colitis ulcerosa Vereinigung)

C. Groß (Berlin), S. In der Smitten (Berlin), B. Kaltz (Berlin), I. Kanbach (Berlin)

- DGAV (Deutsche Gesellschaft für Allgemein- und Viszeralchirurgie)

A. Kroesen (Köln), S. Fichtner-Feigl (Freiburg), M. Kreis (Berlin),

P. Kienle (Mannheim), E. Rijcken (Münster)

- DGCH (Deutsche Gesellschaft für Chirurgie)

A. Kroesen (Köln), S. Fichtner-Feigl (Freiburg), M. Kreis (Berlin)

- DGK (Deutsche e. V. Gesellschaft für Koloproktologie)

A. Kroesen (Köln), S. Fichtner-Feigl (Freiburg), M. Kreis (Berlin),

P. Kienle (Mannheim), E. Rijcken (Münster)

- DGEM (Deutsche Gesellschaft für Ernährungsmedizin)

J. Ockenga (Bremen)

- DGIM (Deutsche Gesellschaft für Innere Medizin)

A. Stallmach (Jena)

- KN-CED (Kompetenznetz Darmerkrankungen)

B. Bokemeyer (Minden)
- DRG (Deutsche Röntgengesellschaft)

E. Schnoy (Augsburg), A. Schreyer (Brandenburg an der Havel)

- GPGE (Gesellschaft für pädiatrische Gastroenterologie und Ernährung)

P. Bufler (Berlin), J. Däbritz (Rostock), J. de Laffolie (Gießen),

K. Keller (Wiesbaden), S. Koletzko (München)

- FACED (Fachangestellte für chronisch entzündliche Darmerkrankungen)

P. Hartmann (Minden), J. Zemke (Minden)

Die Deutsche Gesellschaft für Allgemeinmedizin und Familienmedizin (DEGAM) wurde bei den Leitlinienaktualisierungen Morbus Crohn und Colitis ulcerosa zur Mitarbeit an den Leitlinien eingeladen, konnte das Leitlinienvorhaben aber aufgrund personeller Engpässe nicht unterstützen. Daher wurde die DEGAM bei dem vorliegenden Addendum nicht beteiligt.

\section{Geltungsbereich und Zweck}

Die Infektion mit dem neuen SARS-CoV-2-Virus führt zu einem bisher unbekannten Krankheitsbild, das als COVID-19 (COrona VIrus Disease-2019) bezeichnet wird und erstmals in der Region Hubei in China beschrieben wurde. Die Infektion entwickelte sich im Januar in China zur Epidemie, wurde am 30.01.2020 durch die WHO zur internationalen Gesundheitsnotlage und schließlich am 11.03.2020 zur Pandemie erklärt [1]. Am 28.01.2020 wurde über die erste Infektion in Deutschland berichtet. Laut der Risikobewertung des Robert Koch-Instituts (RKI) wurde am 26.05.2020 die Gefahr für die Gesundheit der Bevölkerung in Deutschland immer noch als hoch, für besondere Risikogruppen als sehr hoch eingeschätzt. In Deutschland sind etwa 450000 Einwohner an einer chronisch entzündlichen Darmerkrankung (CED) erkrankt. Für die meisten Patienten beginnt die Erkrankung während der Schulzeit oder der Berufsausbildung und dauert während des gesamten Lebens an. Neben dem persönlichen Leiden verursacht die Erkrankung beträchtliche Kosten für die Gesellschaft. Für Patienten mit Morbus Crohn bzw. Colitis ulcerosa, die insgesamt zu 33-66\% bzw. 11-29\% mit immunsuppressiv wirkenden Medikamenten einschließlich der Biologika behandelt werden [2, 3], aber auch für die in die Behandlung eingebundenen Personen des Gesundheitssystems stellen sich vor dem Hintergrund der COVID-19-Pandemie viele Fragen.

\section{Zielorientierung der Leitlinie}

Vor diesem Hintergrund ist es das Ziel der Leitlinie, Empfehlungen auszusprechen und Handlungskorridore zu beschreiben. Dabei soll diese Leitlinie in der hausärztlichen, internistischen, chirurgischen, pädiatrischen und gastroenterologischen Praxis einfach anwendbar sein. Die Behandlung besonders schwerer oder komplizierter Fälle kann durch diese Leitlinie nicht abgebildet werden. Betrachtet werden in der Leitlinie Patienten jeden Alters mit einer CED. 


\section{Versorgungsbereich}

Ambulant und stationär: hausärztlich, pädiatrisch, internistisch, chirurgisch und gastroenterologisch.

\section{Anwenderzielgruppe/Adressaten}

Die Leitlinie richtet sich an alle an der Diagnostik und Therapie von Patienten mit CED beteiligten Berufsgruppen (Internisten, Allgemein- und Viszeralchirurgen, Gastroenterologen, Pathologen, Fachassistenz (ED), zudem Betroffene, Angehörige. Sie dient zur Information für Allgemeinmediziner, Kinder- und Jugendmediziner und Leistungserbringer (Krankenkassen, Rentenversicherungsträger).

\section{Zusammensetzung der Leitliniengruppe/Beteiligung von Interessengruppen/methodisches Vorgehen}

Die Leitung des Projekts erfolgte durch vier Hauptkoordinatoren (Axel Dignaß, (Frankfurt), Torsten Kucharzik (Lüneburg), Andreas Stallmach (Jena) und Andreas Sturm (Berlin). Zur Erstellung des Addendums der bestehenden Leitlinie zum Morbus Crohn, die sich gegenwärtig in der Aktualisierungsphase befindet, und zur Colitis ulcerosa [4] wurden universitäre und nichtuniversitäre Ärzte, Klinikärzte und niedergelassene Ärzte aus dem Kreis aller Teilnehmer der beiden Leitlinien eingeladen.

Das Leitlinienaddendum enthält insgesamt 23 Empfehlungen. - Tab. 2 listet die 10 Kernaussagen auf. Insgesamt basiert das Leitlinienaddendum auf einem Konsensus einer interdisziplinären Expertengruppe. Vorab wurden die Themengebiete der einzelnen Kapitel für die aktuellen und praxisnahen Empfehlungen in einer virtuellen Konferenz formuliert und anschließend in einer ersten Delphirunde von der Leitliniengruppe abgestimmt und durch Autorenteams bearbeitet. Dazu wurde die den Kernaussagen zugrunde liegende Literatur basierend auf der aktuellen Datenlage nach den Richtlinien des Oxford Centre for Evidence-based Medicine - Levels of Evidence bewertet und daraus eine Empfehlungsstärke abgeleitet. [5] Die Kernaussagen wurden den AWMFVorgaben entsprechend mit einer Formulierung gemäß ihrer Empfehlungsstärke versehen. Eine starke Empfehlung entspricht in der Formulierung einem „soll“, eine Empfehlung einem „sollte“ und eine offene Empfehlung einem „kann“. Alle Empfehlungen, bis auf die Empfehlung 3.2, wurden in einer zweiten Delphirunde final von der Leitliniengruppe abgestimmt und konsentiert. Die Empfehlung 3.2 wurde aufgrund der Kommentare erneut überarbeitet, abgestimmt und konsentiert. Die Formulierung der Kernaussagen wurden basierend auf diesem Expertenkonsens bei einer Zustimmung von $>95 \%$ aller 68 Experten als starker Konsens, bei $75-95 \%$ als Konsens, bei $>50-75 \%$ als mehrheitliche Zustimmung und bei $<50 \%$ als kein Konsens gewertet (siehe - Tab. 1).

Da für das Management von CED-Patienten mit COVID-19 keine randomisierten Studien gemäß der Evidenzlevel 1 oder 2 existieren, besteht insgesamt nur eine schwache Evidenz für alle Empfehlungen. Zu fast allen Themenbereichen stehen nur Beobachtungsstudien mit teils kleinen Fallzahlen und naturgemäß kurzen Beobachtungszeiträumen, Fallberichte, Expertenmeinungen oder Erfahrungen mit verwandten viralen Erregern zur Verfügung.
- Tab. 1 Einteilung der Konsensstärke.

\begin{tabular}{|l|l|}
\hline Konsens & \%-Zustimmung \\
\hline starker Konsens & $>95$ \\
\hline Konsens & $>75-95$ \\
\hline mehrheitliche Zustimmung & $>50-75$ \\
\hline kein Konsens & $<50$ \\
\hline
\end{tabular}

Hinzu kommt, dass eine objektive Bewertung kaum zu erheben ist und wesentlich durch die Erfahrungen der Experten beeinflusst wird. Die bislang zu diesen Fragestellungen vorliegenden, meist epidemiologischen Studien hinsichtlich der untersuchten Patientenkollektive und Zielparameter sind sehr uneinheitlich. Vor diesem Hintergrund ist es den Autoren wichtig zu betonen, dass Handlungsempfehlungen meist auf persönlichen Erfahrungen und Analogieschlüssen zu anderen Krankheitsbildern beruhen. Unter Berücksichtigung der zahlreichen Fragen der behandelnden Ärztinnen und Ärzte, aber auch der Sorgen und Ängste der Patienten, die zu ungünstigen Entscheidungen, wie z. B. dem Absetzen der Medikation führen könnten, erscheint dieses Leitlinienaddendum aber gerade deshalb dringend notwendig.

\section{Repräsentativität der Leitliniengruppe/Beteiligung von Patienten}

Die Erstellung des Addendums erfolgte unter direkter Mitarbeit mehrerer Vertreter der Deutschen Morbus Crohn/Colitis ulcerosa Vereinigung (DCCV) e. V.

\section{Externe Begutachtung und Verabschiedung}

\section{Verabschiedung durch die Vorstände der heraus- gebenden Fachgesellschaften/Organisationen}

Im Anschluss an den Peer-Review-Prozess wurde die vollständige Leitlinie von den Vorständen aller beteiligten Fachgesellschaften begutachtet und konsentiert.

Darüber hinaus stand die Leitlinie für eine Woche als Konsultationsfassung zur Kommentierung auf der DGVS- und der AWMFWebsite zur Verfügung. Alle Änderungsvorschläge wurden gesichtet und ggf. eingearbeitet.

\section{Redaktionelle Unabhängigkeit und Finanzierung der Leitlinie}

Die Nutzung der CGS-Plattform wurde durch die DGVS finanziert. Eine finanzielle Beteiligung Dritter erfolgte nicht. Alle Mandatsträger und Experten arbeiteten ausschließlich ehrenamtlich.

\section{Darlegung von und Umgang mit Interessenkonflikten}

Im Einklang mit dem AWMF-Regelwerk zum Umgang mit Interessenkonflikten haben vor Beginn der Delphirunde alle Teilnehmer Erklärungen auf dem entsprechenden AWMF-Formular abgegeben. Die Interessenkonflikte wurden von den Koordinatoren der Leitlinie 
- Tab.2 Zentrale Empfehlungen des Addendums (hier die 10 wichtigsten Empfehlungen).

\begin{tabular}{|c|c|c|}
\hline Nr. & Empfehlung & Zustimmungsgrad \\
\hline 1 & $\begin{array}{l}\text { In dieser S2k-Leitlinie besteht insgesamt eine nur schwache Evidenz für die Empfehlungen. Handlungsempfeh- } \\
\text { lungen basieren meist auf persönlichen Erfahrungen und Analogieschlüssen zu anderen Krankheitsbildern. } \\
\text { Notwendige Entscheidungen zur Anpassung der Therapie (medikamentös oder operativ) sollten somit immer } \\
\text { der aktuellen Datenlage angepasst werden. }\end{array}$ & starker Konsens (98\%) \\
\hline 1.1 & $\begin{array}{l}\text { Patienten mit einer CED haben generell kein erhöhtes Risiko für eine Infektion mit SARS-CoV-2. Dennoch } \\
\text { sollten Patienten sorgfältig individuelle Schutzmaßnahmen ergreifen. }\end{array}$ & starker Konsens (95\%) \\
\hline 1.2 & $\begin{array}{l}\text { Patienten mit einer CED und einer immunsuppressiven Therapie haben ein erhöhtes Risiko für eine SARS-CoV-2- } \\
\text { Infektion und sollten deshalb sorgfältig individuelle Schutzmaßnahmen umsetzen. Der Grad der Risikoerhö- } \\
\text { hung scheint dabei für einzelne Immunsuppressiva unterschiedlich zu sein. }\end{array}$ & Konsens (86\%) \\
\hline 1.3 & $\begin{array}{l}\text { Patienten mit einer CED und einer SARS-CoV-2-Infektion haben unter bestimmten Bedingungen (Komorbiditäten/ } \\
\text { Risikofaktoren) ein erhöhtes Risiko für einen schweren Verlauf der COVID-19-Erkrankung. Diese Patienten sollten } \\
\text { sorgfältig bezüglich einer raschen Verschlechterung ihrer Erkrankung überwacht werden. }\end{array}$ & Konsens (93\%) \\
\hline 2.1 & $\begin{array}{l}\text { Während der Pandemie sollten Patientenvorstellungen in Einrichtungen des Gesundheitswesens restriktiv } \\
\text { erfolgen. CED-Sprechstunden sollten unter Berücksichtigung der Dringlichkeit der Vorstellung und unter } \\
\text { Optimierung der Infektionsschutzmaßnahmen wie räumlicher Distanzierung und nach Ausnutzen von } \\
\text { Möglichkeiten der Telemedizin fortgeführt werden. }\end{array}$ & Konsens (86\%) \\
\hline 2.2 & $\begin{array}{l}\text { Während der Pandemie sollten sämtliche endoskopischen Untersuchungen unter besonderen Schutzmaß- } \\
\text { nahmen stattfinden. Das Ausmaß der Schutzmaßnahmen sollte risikoadaptiert erfolgen. }\end{array}$ & starker Konsens (98\%) \\
\hline 3.1 & $\begin{array}{l}\text { Patienten mit einer CED und einer immunsuppressiven Therapie haben generell kein erhöhtes Risiko für } \\
\text { einen schweren Verlauf einer SARS-CoV-2-Infektion. Eine immunsuppressive Therapie sollte deshalb bei einer } \\
\text { leichten bis moderaten COVID-19-Erkrankung nicht reduziert werden. Eine Ausnahme bilden die länger dau- } \\
\text { ernde Therapie mit systemischen Steroiden insbesondere in Dosierungen größer } 20 \text { mg Prednisonäquivalent/ } \\
\text { Tag. Diese sollte daher möglichst vermieden oder, soweit klinisch vertretbar, reduziert und beendet werden. }\end{array}$ & Konsens (84\%) \\
\hline 3.2 & $\begin{array}{l}\text { Bei Patienten mit schwerer COVID-19-Erkrankung sollte die Therapie mit Thiopurinen, Methotrexat und } \\
\text { Tofacitinib pausiert und nach Überwinden der Infektion wieder aufgenommen werden. }\end{array}$ & Konsens (94\%) \\
\hline 3.4 & $\begin{array}{l}\text { Während der SARS-CoV-2-Pandemie sollte eine Biologikatherapie mit zu erwartendem raschem Wirkeintritt } \\
\text { gegenüber einer hochdosierten systemischen Steroidtherapie im akuten Schub bevorzugt eingesetzt werden. }\end{array}$ & Konsens (85\%) \\
\hline 4.3 & $\begin{array}{l}\text { Hospitalisierte Patienten mit einer CED und COVID-19-Erkrankung sollten mindestens eine Thrombosepro- } \\
\text { phylaxe erhalten. Bei ambulanten COVID-19-erkrankten CED-Patienten sollte entsprechend ihres individuel- } \\
\text { len Risikoprofils und ihrer Begleitmedikation die Entscheidung für eine Thromboseprophylaxe großzügig } \\
\text { getroffen werden. }\end{array}$ & starker Konsens (95\%) \\
\hline
\end{tabular}

und Frau Lynen gesichtet und der Leitliniengruppe vor Beginn der Delphirunde präsentiert. Die Interessenkonflikte wurden zunächst auf thematischen Bezug zur Leitlinie gesichtet und gemäß den AWMF-Kriterien als keine, gering, moderat oder hoch bezüglich der individuellen Empfehlung kategorisiert. Die Mandatsträger der Leitlinie gaben insgesamt eine Vielzahl von potenziellen Interessenkonflikten an. Nach Einschätzung der Leitliniengruppe stellen die interdisziplinäre Besetzung der Leitliniengruppe (einschließlich stimmberechtigter Patientenvertreter) und die selektive Literaturrecherche und deren Bewertung eine wichtige Maßnahme zum Ausgleich dieser potenziellen Interessenkonflikte dar. Mandatsträger mit personenbezogenen Zuwendungen (Zugehörigkeit zu Advisory Boards, Gutachter- und Vortragstätigkeit) wurden daher nach kritischer Bewertung durch die Leitliniengruppe nicht von den Abstimmungen ausgeschlossen, wenn die Art der Zuwendungen nicht einseitig (z. B. Zugehörigkeit zu mehreren Advisory Boards) und die wissenschaftliche Expertise nicht verzichtbar war. Finanzielle Zuwendungen, die ausschließlich wissenschaftlichen Institutionen zugeordnet werden konnten, führten nicht zu einer Stimmenthaltung (Drittmittel, Studienbeteiligung). Mandatsträger, deren
Interessenkonflikte nicht vorlagen, oder Mandatsträger mit Eigentümerinteressen (z. B. Patente, Aktienbesitz, Firmenzugehörigkeit) erhielten kein Stimmrecht. Nach Überprüfung aller Interessenkonflikte wurden keine Mandatsträger ausgeschlossen. Die Interessenkonflikte sind im Anhang veröffentlicht.

\section{Verbreitung und Implementierung}

\section{Konzept zur Verbreitung und Implementierung}

Das Addendum wird in der Zeitschrift für Gastroenterologie, im AWMF-Leitlinienportal (www.awmf.de) und auf der Homepage der DGVS (www.dgvs.de) veröffentlicht. Eine englische Übersetzung soll zur Verfügung gestellt werden. Für die Information von Laien oder Patienten wird auf die Homepage der Deutschen Morbus Crohn/Colitis ulcerosa Vereinigung - DCCV - e. V. (https://www.dccv.de/betroffene-angehoerige/leben-mit-einerced/infektionskrankheitenimpfen/coronavirus/) verwiesen. 


\section{Gültigkeitsdauer und Aktualisierungsverfahren}

Die Gültigkeit des Addendums orientiert sich an der Gültigkeit der S3-Leitlinie Colitis ulcerosa und wird daher auf 3 Jahre geschätzt. Eine Überarbeitung wird durch den Leitlinienbeauftragten der DGVS initiiert werden. Sollte es zwischenzeitlich wichtige Neuerungen in der Therapie der COVID-19-Erkrankung geben, die eine kurzfristige Aktualisierung notwendig erscheinen lassen, entscheiden die Koordinatoren (A. Stallmach, A. Sturm, T. Kucharzik, A. Dignaß) gemeinsam mit der Leitlinienbeauftragten der DGVS (B. Siegmund) über die Notwendigkeit und die evtl. Inhalte einer Aktualisierung. Diese soll dann online im Leitlinienportal der AWMF und auf der Homepage der DGVS veröffentlicht werden.

Der Informationsstand zur SARS-CoV2-Infektion und zu COVID-19 kann sich rasch ändern; täglich erscheinen dazu neue wissenschaftliche Publikationen. Auch die offiziellen Handlungsempfehlungen des Robert Koch-Instituts (RKI: https://www.rki. de/DE/Content/InfAZ/N/Neuartiges_Coronavirus/nCoV_node. html), der Weltgesundheitsorganisation (WHO: https://www. who.int/emergencies/diseases/novel-coronavirus-2019) und der DGVS (https://www.dgvs.de/COVID-19/) werden kontinuierlich überarbeitet und sollten im Bedarfsfall in ihrer aktuellen Fassung im Internet eingesehen werden.

\section{Redaktioneller Hinweis}

\section{Geschlechtsneutrale Formulierung}

Ausschließlich zum Zweck der besseren Lesbarkeit wird auf die geschlechtsspezifische Schreibweise verzichtet. Alle personenbezogenen Bezeichnungen in diesem Dokument sind somit geschlechtsneutral zu verstehen.

\section{Partizipative Entscheidungsfindung}

Alle Empfehlungen der Leitlinie sollten im Sinne einer partizipativen Entscheidungsfindung von Arzt und Patient und ggf. den Angehörigen bewertet und ggf. umgesetzt werden.

\section{Einleitung}

Die Infektion mit dem neuen SARS-CoV-2-Virus führt zu einem bisher unbekannten Krankheitsbild, das als COVID-19 (COrona VIrus Disease-2019) bezeichnet wird und erstmals in der Region Hubei in China beschrieben wurde [6, 7]. Die Infektion wurde durch die WHO am 11.03.2020 zur Pandemie erklärt [1]. Das SARS-CoV-2-Virus, das bisher Menschen nicht infizierte, gehört zur großen Gruppe der Coronaviren, die Erkrankungen von einer normalen Erkältung bis zu schweren Krankheitsverläufen verursachen können. Verwandt mit dem SARS-CoV-2-Virus sind die Viren, die das Krankheitsbild des SARS (Schweres Akutes Respiratorisches Syndrom) und des MERS (Middle East Respiratory Syndrome) verursachen. Trotz der durch die Landesregierungen verordneten Maßnahmen der gesellschaftlichen Ausgangs- und Kontaktsperre und anderer Schutzmaßnahmen stieg die Rate der Patienten mit COVID-19-Erkrankungen kontinuierlich an; in
Deutschland erkrankten mehr als 173000 Menschen, und 7881 Menschen verstarben an der Infektion (Stand 16.05.2020, RKI).[8]

Vor allem für Menschen mit CED stellen sich viele Fragen. Hilfreich wären evidenzbasierte Handlungsempfehlungen; aufgrund der zurzeit unzureichenden Datenlage mit meist Registerstudien basieren diese vor allem auf Expertenmeinungen und Analogien zu anderen infektiösen Krankheitsbildern. Kritisch anzumerken ist aber, dass fehlende Evidenzen nicht zwangsläufig durch einen Konsensusprozess zu ersetzen sind. Selbst unter Experten unterliegt eine solche Meinungsbildung kurzlebigen Zeitströmungen, und nicht jede Wissenslücke ist grundsätzlich füllbar. Insgesamt erscheint aber angesichts der Sorgen und Ängste der Patienten, die zu folgereichen Entscheidungen, wie z. B. dem Absetzen der Medikation führen können, und der offenen Fragen bei behandelnden Ärzten diese S2k-Leitlinie dringend notwendig.

\section{EMPFEHLUNG 1}

In dieser S2k-Leitlinie besteht insgesamt eine nur schwache Evidenz für die Empfehlungen. Handlungsempfehlungen basieren meist auf persönlichen Erfahrungen, Daten aus publizierten Fallserien und Registern sowie Analogieschlüssen zu anderen Krankheitsbildern. Notwendige Entscheidungen zur Anpassung der Therapie (medikamentös oder operativ) sollten somit immer der aktuellen Datenlage angepasst werden. Empfehlungsgrad: EL5/Zustimmungsgrad: starker Konsens (98\%)

Die klinischen Manifestationen des Krankheitsbildes sind unspezifisch und hoch variabel. Sie reichen von asymptomatischen, milden Krankheitsbildern bis hin zu schweren Lungenerkrankungen mit ARDS, Multiorganversagen und Tod. Allgemeingültige Aussagen zum „typischen" Krankheitsverlauf lassen sich nicht machen. Die kumuliert in China erfassten Patienten ( $n=55$ 924) berichteten am häufigsten über Fieber und Husten. Rund $80 \%$ der Erkrankungen verliefen mild bis moderat. Als „mild“ werden Krankheitsverläufe ohne klinische oder radiologische Zeichen einer Pneumonie bezeichnet, „moderat“ Erkrankte haben eine leichte Pneumonie, die sich nach radiologischen Kriterien auf weniger als die Hälfte der Lunge ausdehnt; die Patienten geben keine Atemnot an und weisen eine Sauerstoffsättigung im Blut von über $93 \%$ auf [9]. Bei 14\% der Patienten verlief die Erkrankung schwer (mit Atemnot, Sauerstoffsättigung unter $94 \%$ oder Lungeninfiltraten in mehr als der Hälfte der Lunge), aber nicht lebensbedrohlich. In $6 \%$ der Fälle war der klinische Verlauf kritisch bis lebensbedrohlich (Lungenversagen, septischer Schock oder multiples Organversagen, ausgedehnte Thrombembolien). In einer Kohorte von 651 Patienten wiesen 74 (11,4\%) gastrointestinale Symptome (Übelkeit, Erbrechen und Diarrhoe) auf, die auch den Symptomen eines akuten Schubs bei bekannter CED entsprechen können [10]. Bei 40 Patienten mit gesicherter CED und begleitender COVID-19Erkrankung wurden Diarrhoen bei $21 \%$ beschrieben [11]. Daten aus dem weltweiten IO IBD-Register, in dem Mitte Mai 2020 über 1300 Patienten mit bekannter CED und nachgewiesener COVIDErkrankung dokumentiert werden, beschreiben, dass sich zum Zeitpunkt der COVID-19-Erkrankung 59\% der Patienten in Remission be- 


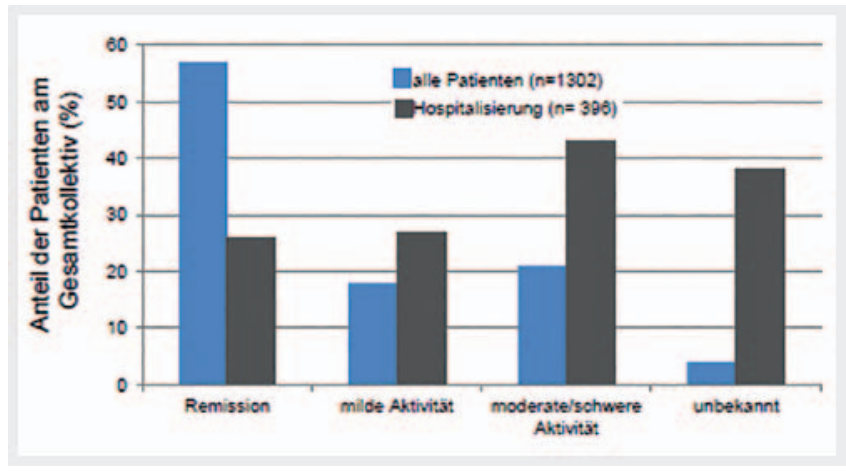

- Abb. 1 Krankheitsaktivität bei Patienten mit CED und COVID-19Erkrankung. Der überwiegende Teil der CED Patienten erkrankt an COVID-19 in einer Phase der Remission. Patienten mit moderater bis schwerer CED-Krankheitsaktivität werden häufiger hospitalisiert [12].

fanden, $18 \%$ wiesen eine milde und $20 \%$ eine moderate Aktivität der Grunderkrankung auf. Die Mehrzahl der Patienten wurde weiter ambulant betreut; tendenziell werden mehr Patienten mit CED und moderater bzw. hoher Krankheitsaktivität bei COVID-19 hospitalisiert (siehe auch - Abb. 1) [12]:

Der Pathomechanismus der gastrointestinalen Symptomatik bei COVID-19-Erkrankung ist unklar. Das SARS-CoV-2-Virus bindet an den ACE2-Rezeptor, der im gesamten Gastrointestinaltrakt, d. h. vom Ösophagus bis um Rektum, exprimiert ist, und dringt so in die Zelle ein. Diese direkten Schäden aufgrund invadierender Viren können so stark ausgeprägt sein, dass sich Patienten mit einer hämorrhagischen Colitis präsentieren [13]. Auch gibt es Patienten, bei denen der Virusnachweis im Rachenabstrich oder Sputum negativ war, die aber einen positiven Virusnachweis im Stuhl hatten [14]. Möglicherweise spielt eine sekundäre Malabsorption aufgrund geschädigter Enterozyten eine Rolle [15].

Außerhalb von Wuhan/Hubei gibt es Beobachtungen, dass der Anteil milder Verläufe höher als $80 \%$ ist. Naturgemäß hängt die Höhe einer Prozentangabe von der Stichprobengröße und dem Umstand ab, wie Patienten identifiziert wurden (Massenscreening oder symptomorientiertes Vorgehen). In allen Publikationen zeigt sich jedoch eine ähnliche Häufigkeitsverteilung der Symptome [16-19] (siehe $>$ Abb. 2).

\section{Kapitel 1: Risiko für eine COVID-19-Erkrankung bei Patienten mit chronisch entzündlichen Darmerkrankungen}

\section{EMPFEHLUNG 1.1}

Patienten mit einer CED haben generell kein erhöhtes Risiko für eine Infektion mit SARS-CoV-2. Dennoch sollten Patienten sorgfältig individuelle Schutzmaßnahmen ergreifen. Empfehlungsgrad: EL5/Zustimmungsgrad: starker Konsens (95\%)

Systematische Befragungen von Patienten mit CED belegen, dass diese sich große Sorgen machen, dass bei ihnen ein erhöhtes Risiko

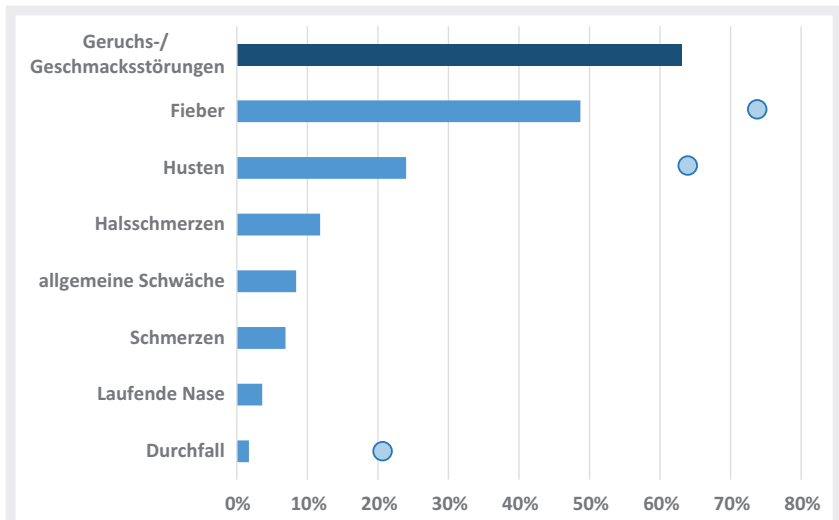

- Abb. 2 Symptome von Patienten mit COVID-19. (Daten des ECDC, 2020: „The European Surveillance System (TESSy) [20]. Die Angaben zu den Beeinträchtigungen des Geruchs-/Geschmackssinns stammen aus verschiedenen europäischen Studien [21]. Symptome einer Fallserie von CED-Patienten sind als Punkte dargestellt [11].

für eine Infektion mit SARS-CoV-2 besteht [22]. Erste epidemiologische Daten zeigen, dass CED-Patienten kein erhöhtes Risiko für eine COVID-19-Erkrankung haben. So wurde aus der IBD Elite Union, dem siebtgrößten CED-Zentrum in China, das mehr als 20000 Patienten betreut, kein Fall einer COVID-19-Erkrankung bei CED-Patienten beschrieben [23]. In Italien wurde in einer systematisch betreuten Kohorte von 522 Patienten kein Fall identifiziert [24]; eine größere Kohorte aus Madrid mit 1918 CED-Patienten weist 12 COVID-19-Infektionen aus; dieses entspricht einer Inzidenz von 6,1 COVID-19-Infektionen auf 1000 CED-Patienten bei einer Inzidenz in der Gesamtbevölkerung von 6,6/1000 [25]. In einer noch größeren Kohorte aus Nancy (Frankreich) und Mailand (Italien) mit 6000 Patienten wird über eine Inzidenz von 2,5/1000 CED-Patienten bei einer Inzidenz von 1,7/1000 in der Normalbevölkerung berichtet [26]. Die Schwäche aller dieser Untersuchungen ist jedoch, dass nur symptomatische Patienten erfasst wurden und nicht die Gesamtkohorte getestet wurde.

Das selektive Testen von nur symptomatischen Patienten erklärt zumindest partiell, dass der Anteil der Kinder und Jugendlichen an den COVID-19-Erkrankten weltweit sehr niedrig ist. Obwohl Kinder im Allgemeinen anfälliger für Infektionen sind, weisen inzwischen zahlreiche Untersuchungen darauf hin, dass es sich bei der SARS-CoV-19 Infektion anders verhält. Kinder scheinen sich seltener zu infizieren $[27,28]$ und haben im Fall einer Infektion meist einen milden Krankheitsverlauf. Die im Mai im Deutschen Ärzteblatt publizierten Zahlen aus Deutschland geben einen Anteil von 3 \% Kinder und Jugendliche aller dem RKI gemeldeten Patienten mit nachgewiesener SARS-CoV-2-Infektion an, obwohl diese Altersklasse $13 \%$ der Gesamtpopulation entspricht. Von 128 stationären pädiatrischen Patienten waren zwei Drittel Säuglinge und Kleinkinder und nur ein Drittel Schulkinder und Jugendliche. Die meisten Patienten waren nicht wegen COVID-19 hospitalisiert, $17 \%$ zeigten keine COVID-typischen Symptome, nur $5 \%$ wurden beatmet und ein Kind verstarb [29]. Ein ähnliches Bild mit Hinweis auf einen leichteren Krankheitsverlauf beim Kind im Vergleich zum Erwachsenen, ergibt sich bei den 1302 im Secure-IBD-Register gemeldeten CED-Patienten mit COVID-19; 
61 (4,7\%) waren jünger als 20 Jahre, davon mussten nur vier stationär aufgenommen werden, keiner wurde intensivpflichtig [12]. Die IBD-Arbeitsgruppe der ESPGHAN trug die bisherigen Erfahrungen aus Wuhan in China und Süd-Korea zusammen. Von dort wurde von keinem infizierten Kind mit CED berichtet [30]. Von acht in Europa gemeldeten pädiatrischen CED-Patienten mit COVID-19 hatten alle einen milden Verlauf der Infektion, obwohl alle mit Immunsuppressiva behandelt waren [30]. Zusammenfassend scheinen Kinder und Jugendliche mit CED ein geringes Risiko für einen symptomatischen oder schweren Verlauf einer SARSCoV-2-Infektion zu haben. Wie häufig Kinder im Fall einer asymptomatischen Infektion Ausgangspunkt einer Infektionskette sind, konnte unter den Bedingungen der letzten Wochen mit Schließung von Kitas, Kindergärten und Schulen nicht untersucht werden. Nach dem jetzigen Wissensstand können Kinder ohne Komorbiditäten für ein erhöhtes Risiko eines schweren COVID-19-Verlaufs am Schulunterricht teilnehmen, wenn dort die empfohlenen Vorsichtsmaßnahmen eingehalten werden können. Ob die Rückkehr von Klein- und Vorschulkindern, die sich nicht an Hygiene- und Abstandsregeln halten, eine deutliche Risikoerhöhung für eine SARS-CoV-2-Infektion für CED-Patienten oder für an CED erkrankte Geschwister oder Eltern darstellt, kann zum Zeitpunkt der Abfassung der Leitlinie nicht eingeschätzt werden. Letztlich wird die Frage des Risikos für eine SARS-CoV-2-Infektion in den verschiedenen Altersklassen unabhängig von Symptomen erst durch große epidemiologische Untersuchungen bei Einsatz hoch sensitiver und spezifischer serologischer Tests beantwortet werden können.

Für die Bewertung des Risikos für eine SARS-CoV-2-Infektion sind aber auch andere virale Infektionen, die über den oberen Respirationstrakt aufgenommen werden, von besonderem Interesse. So ist ein erhöhtes Risiko für Patienten mit CED, an Influenza zu erkranken bzw. aus diesem Grund hospitalisiert zu werden, beschrieben [31]. Weiterhin beschreiben Ko und Mitarbeiter für CED-Patienten im ersten Krankheitsjahr eine Hospitalisierung aufgrund von Infektionen bei 7,9\%; dabei ist mehr als 1/3 durch Infektionen des Respirationstrakts bedingt [32].

Um das Risiko für eine COVID-19-Erkrankung zu minimieren, sind als wichtigste Maßnahmen hygienische Händedesinfektionen, das Einhalten eines Abstandes ( $\geq 1,5-2 \mathrm{~m}$ ) zu anderen Personen, die Eingrenzung sozialer Kontakte und das Verzichten auf Reisen zu nennen. Die konsequente Umsetzung dieser Empfehlungen wird auch durch ein aktuelles Positionspapier der ECCO betont [33]. Aufgrund einer Studie aus China wird spekuliert, dass bei konsequenter Umsetzung der Schutzmaßnahmen CEDPatienten vor einer COVID-19-Erkrankung geschützt werden können [34]. Verschiedene Studien zeigen, dass durch Händewaschen unter Verwendung von Seife (mindestens 15 Sekunden) die Häufigkeit von grippeähnlichen Infektionen signifikant reduziert wird [35]. Eine weitere - allerdings kontrovers diskutierte - Maßnahme ist das Tragen einer Mund-Nasen-Bedeckung (MNB). Das RKI empfiehlt ein generelles Tragen der MNB in bestimmten Situationen im öffentlichen Raum als einen weiteren Baustein, um Risikogruppen zu schützen und den Infektionsdruck und damit die Ausbreitungsgeschwindigkeit von SARS-CoV-2 in der Bevölkerung zu reduzieren. Diese Empfehlung beruht auf einer Neubewertung der zunehmenden Evidenz, dass ein hoher Anteil von Übertragun- gen bei hoher Viruslast unbemerkt bereits 2-3 Tage vor dem Auftreten von Krankheitssymptomen erfolgen kann.[36, 37]. Zu betonen ist, dass der Schutzeffekt nicht auf die Protektion des MNB-Trägers selbst (als eines potenziell gefährdeten Patienten), sondern auf den Schutz des Kollektivs vor dem potenziell infektiösen MNB-Träger abzielt. Von Patienten wird aber auch immer wieder hinterfragt, ob z. B. spezielle filtrierende Halbmasken (sogenannte FFP2-Masken) notwendig oder hilfreich sind, um einen individuellen Schutz zu erreichen. Dazu gibt es keine belastbaren Daten. Wichtig in dem Zusammenhang erscheint aber die Beobachtung, dass bei Krankenhauspersonal, das einen unerkannt an COVID-19 erkrankten Patienten auf der Intensivstation versorgt hatte, innerhalb eines 14-tägigen Nachbeobachtungsintervalls keine Infektionen aufgefallen sind. Dabei wurde in $85 \%$ der potenziellen Infektionsexpositionen lediglich eine chirurgische MundNasen-Maske getragen [38]. Die Verwendung von FFP2-Masken bei CED-Patienten ohne besondere Risikoexposition zum Eigenschutz ist somit nicht nachweisbar indiziert.

\section{EMPFEHLUNG 1.2}

Patienten mit einer CED und einer immunsuppressiven Therapie haben ein erhöhtes Risiko für eine SARS-CoV-2-Infektion und sollten deshalb sorgfältig individuelle Schutzmaßnahmen umsetzen. Der Grad der Risikoerhöhung scheint dabei für einzelne Immunsuppressiva unterschiedlich zu sein.

Empfehlungsgrad EL5/Zustimmungsgrad: Konsens (86\%)

Zur tatsächlichen Höhe des Infektionsrisikos fehlen spezifische epidemiologische Daten für Patienten mit CED mit immunsuppressiver Therapie, die eine konkrete Risikoabschätzung für die Infektion mit SARS-CoV-2 möglich machen. Die Zahl der Patienten mit Biologikatherapie bzw. klassischer Immunsuppression in den oben erwähnten Kohorten ist niedrig; teilweise wurde diese mit Beginn der Pandemie abgesetzt; somit sind die Fallzahlen zu niedrig, um verlässliche Aussagen zu machen. Ohne Zweifel ist insgesamt das Risiko für bakterielle und virale Infektionen bei Patienten mit einer immunsuppressiven Therapie bzw. einer Steroidmedikation signifikant erhöht; diese bedingen auch eine erhöhte Mortalität[39]. Epidemiologische Studien aus der Lombardei in Italien zu 1193 Patienten mit Psoriasis und Biologika ergeben ein erhöhtes Risiko für eine Infektion, aber nicht für einen schweren Verlauf im Sinne einer Notwendigkeit für eine intensivmedizinische Betreuung oder Tod (siehe auch > Tab. 3) [40].

Seit langer Zeit ist bekannt, dass eine Steroidmedikation, insbesondere in Dosierungen über $20 \mathrm{mg} /$ Tag Prednisolonäquavivalent, das Risiko für opportunistische Infektionen einschließlich Influenzainfektionen und schwerer Pneumonien erhöht und eine signifikant gesteigerte Häufigkeit von Hospitalisierungen und eine erhöhte Mortalität bedingt [41-44]. In einer großen Fallkontrollstudie mit 140.000 Patienten mit CED war eine Steroidmedikation ein unabhängiger Risikofaktor für eine Influenzainfektion (Odds Ratio, 1.22; 95 \%-KI: 1,08-1,38) [31]. Somit muss eine Steroidmedikation, insbesondere in höheren Dosierungen, als ein Risikofaktor für eine COVID-19-Erkrankung angenommen werden. 
- Tab. 3 Risiko für SARS-CoV-2-Infektionen bei Patienten mit Psoriasis und Biologikatherapie. Angegeben ist das relative Risiko im Vergleich zur Gesamtpopulation [40].

\begin{tabular}{|l|l|l|l|}
\hline Parameter & Risiko & $\begin{array}{l}\text { 95\%-Vertrau- } \\
\text { ensintervall }\end{array}$ & P-Wert \\
\hline Positiver SARS-CoV-2-Test & 3,43 & $2,25-5,73$ & $<0,0001$ \\
\hline Quarantäne zu Hause & 9,05 & $5,61-14,61$ & $<0,0001$ \\
\hline Hospitalisierung & 3,59 & $1,49-8,63$ & 0,0044 \\
\hline ITS-Aufnahme & 3,41 & $0,21-54,55$ & 0,3861 \\
\hline Tod & 0,41 & $0,003-6,59$ & 0,5306 \\
\hline
\end{tabular}

Auch die Medikation mit Thiopurinen geht mit einer Risikoerhöhung für virale Infektionen einher [45]. Ob das Risiko für Infektionen mit respiratorischen Viren dabei erhöht ist, ist unklar [46]. Im Vergleich zu einer Thiopurin-Monotherapie ist die Anti-TNFTherapie mit einem niedrigeren Risiko für virale Infektionen (HR: 0,57; $95 \%-K I: 0,38-0,87$ ) assoziiert [47]. Für ein insgesamt erhöhtes Risiko für respiratorische Infektionen unter einer AntiTNF-Monotherapie gibt es keine Hinweise. Allerdings weist die Untersuchung von Kirchgesner et al. ein erhöhtes Risiko für opportunistische virale Infektionen unter einer Anti-TNF-Monotherapie im Vergleich zu nicht exponierten Patienten mit CED auf [47]. Eine COMBO-Therapie aus Anti-TNF-Antikörper plus klassischem Immunsuppressivum (meist Azathioprin/6-Mercaptopurin oder Methotrexat) ist mit einem höheren Risiko für opportunistische Infektionen verbunden als eine Anti-TNF-Monotherapie (Hazard Ratio [HR]: 1,96; $95 \%-K I:$ 1,32-2,91). Dabei sind ältere, multimorbide Patienten besonders gefährdet [48].

Zurzeit werden verschiedene JAK-Inhibitoren vor dem Hintergrund des Zytokinsturms bei Patienten mit schwerer COVID-19-Erkrankung in klinischen Studien getestet (siehe unten). Wichtig ist aber auch, dass in frühen Phasen viraler Infektionen die Interferonmediierte Transkription über die JAK-STAT-Signalkaskaden zur Hochregulation von Genen führt, die die Abtötung von Viren in infizierten Zellen vermitteln [49]. Als Konsequenz kann eine JAKSTAT-Blockade zu einer verminderten interferonvermittelten antiviralen Antwort führen. So sind virale Infektionen (einschließlich Herpes Zoster und Herpes simplex) für $10 \%$ der ambulant erworbenen und $5 \%$ der beatmungsassoziierten Pneumonien bei Patienten auf der Intensivstation ursächlich [50]. Vor diesem Hintergrund sollten JAK-Inhibitoren mit Vorsicht eingesetzt werden [51].

Die Behandlung mit Vedolizumab erhöht das Risiko für Infektion im Respirationstrakt nicht [52]. Auch für Ustekinumab scheint kein erhöhtes Risiko für Infektionen vorzuliegen. So zeigen in einer Übersichtsarbeit zusammengestellte Daten zu Patienten mit Psoriasis-Arthritis [53] bzw. ältere Ergebnisse aus dem PSOLAR-Register insgesamt keine Risikoerhöhung für Ustekinumab im Vergleich zu Basistherapeutika, auch wenn zu der Häufigkeit respiratorischer Infektionen in den Studien nicht dezidiert Stellung genommen wird [53]. - Abb. 3 fasst das Risiko für virale Infektionen in Abhängigkeit von einer medikamentösen Therapie schematisch zusammen.

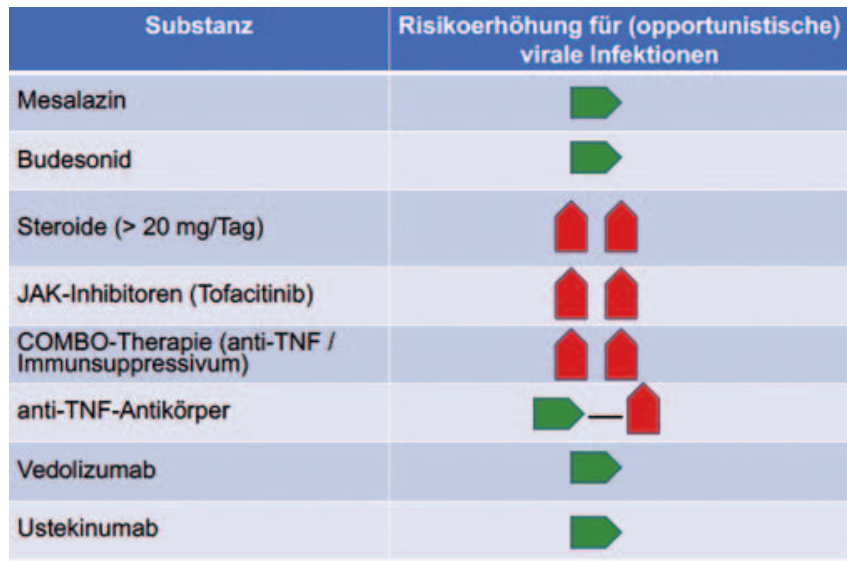

- Abb. 3 Risikoerhöhungen für virale Infektionen bei Patienten mit CED in Abhängigkeit von der medikamentösen Therapie. Modifiziert nach den Empfehlungen der „International Organization for the Study of Inflammatory Bowel Cisease (IOIBD) [54].

\section{EMPFEHLUNG 1.3}

Patienten mit einer CED und einer SARS-CoV-2-Infektion haben unter bestimmten Bedingungen (Komorbiditäten/Risikofaktoren) ein erhöhtes Risiko für einen schweren Verlauf der COVID-19-Erkrankung. Diese Patienten sollten sorgfältig bezüglich einer raschen Verschlechterung ihrer Erkrankung überwacht werden.

Empfehlungsgrad: EL4/Zustimmungsgrad: Konsens (93\%)

Im Gegensatz zu vielen anderen Infektionserkrankungen, bei denen vom Auftreten erster Symptome bis zur klinischen Verschlechterung nur wenige Tage und z. T. nur Stunden vergehen, zeichnet sich die COVID-19-Erkrankung durch einen variablen Verlauf mit z. T. langsam zunehmender Krankheitsschwere, z. T. jedoch auch rasch progredienten Verläufen aus. Die mediane Inkubationszeit von der Exposition bis zum Auftreten erster Symptome beträgt 5-6 Tage bei einer Spannbreite von 2 bis 7 Tagen [55]. Die maximale Inkubationszeit wird vom RKI mit 14 Tagen angenommen. Es gibt jedoch Fallberichte von längeren Inkubationszeiten von bis zu 19 oder 24 Tagen. Somit ist bei vielen Patienten mit COVID-19 die Erkrankung zunächst kompensiert, dann kann aber eine rasche Verschlechterung eintreten. Bekanntlich sind Alter und Vorerkrankungen in hohem Maß miteinander assoziiert; es sind bis jetzt jedoch keine Regressionsanalysen publiziert, die den unabhängigen Einfluss der einzelnen Faktoren beschreiben. Folgende Personen sind für schwere Verläufe besonders gefährdet:

- ältere Personen (mit stetig steigendem Risiko für schwere Verläufe ab etwa 50-60 Jahren)

- Raucher (schwache Evidenz)

- ausgeprägte Adipositas/deutliche Kachexie

- Personen mit Komorbiditäten:

- chronische Lungenerkrankungen (z. B. COPD)

- koronare Herzerkrankung und/oder arterielle Hypertonie 
- Tab. 4 COVID-19-Erkrankungsrisiko medizinischen Personals im Vergleich zu Berufstätigen sowie der Gesamtbevölkerung.

\begin{tabular}{|l|c|c|c|}
\hline & Personenzahl & gemeldete Infektionen & relativer Anteil [\%] \\
\hline med. Personal & 5700000 & 15495 & 0,27 \\
\hline Berufstätige & 45130000 & $107000^{*}$ & 0,23 \\
\hline Gesamtbevölkerung & 83020000 & 159119 & 0,19 \\
\hline * bezogen auf einen 92 \%igen Anteil von Berufstätigen. & & \\
\hline
\end{tabular}

- chronische Lebererkrankungen

- Diabetes mellitus

- maligne Erkrankungen

- Immundefizienz (angeboren oder erworben).

Ohne Zweifel wird bei Patienten mit CED das Risiko für Infektionen bzw. einen schweren Verlauf durch die Medikation beeinflusst („Therapie als Risikofaktor“). Dennoch sind auch die Krankheitsaktivität (OR: 3,35; 95 \%-KI: 1,23-9,23) [45] und eine Malnutrition (OR: 6,26; $95 \%-K I: 1,20-32,78)[56,57]$ signifikante nichtpharmakologische Risikofaktoren. In einer kleineren Observationsstudie aus Italien unter Einschluss von 79 Patienten mit CED und COVID-19-Erkrankung zeigte sich, dass das Alter über 65 Jahre und die Krankheitsaktivität mit der Entwicklung einer schweren COVID-19-Pneumonie bzw. dem Versterben assoziiert waren [58]. Vor diesem Hintergrund sind eine gute Einstellung der Grunderkrankung und der Ausgleich von Ernährungsdefiziten anzustreben. Ob Risikopatienten von einer besonderen Überwachung z. B. durch Verwendung von „CORONAApps“ profitieren, ist unklar und Gegenstand aktueller Studien.

\section{EMPFEHLUNG 1.4}

Bei Patienten mit einer CED und einer immunsuppressiven Therapie, die in Berufen mit einer hohen Zahl sozialer Kontakte zu Menschen mit unbekanntem Risiko für eine SARS-CoV-2-Infektion oder gesicherter COVID-19-Erkrankung arbeiten (z. B. Personen, die im Gesundheitssystem arbeiten), kann ein Wechsel des Arbeitsplatzes vorgenommen werden.

Empfehlungsgrad: EL5/Zustimmungsgrad: Konsens (84\%)

Es gibt bislang keinen eindeutigen Hinweis darauf, dass sowohl pädiatrische als auch erwachsene Patienten mit einer CED insgesamt eine erhöhte Suszeptibilität für eine COVID-19-Erkrankung haben. Hochwahrscheinlich erhöht eine immunsuppressive Therapie das Risiko für eine Infektion, auch wenn möglicherweise die Erkrankung bei Vorbehandlung mit bestimmten Immunsuppressiva milder verläuft (siehe Empfehlung 1.2). Auch hängt das Infektionsrisiko von der Zahl der Kontakte und der Viruslast ab. Für diese Risikoabschätzung ist auch immer die regionale Zahl der SARSCoV-2-Infizierten von Bedeutung. In den aktuellen Hochprävalenzregionen wie z. B. den Stadt- und Landkreisen Heinsberg, Traunstein, Greiz, Zollernalbkreis oder Weiden i. d. OPF ist das Ri- siko für eine Infektion im Rahmen von sozialen Kontakten deutlich höher als in Niedrigprävalenzregionen.

Unter Beachtung der gültigen Hygienevorgaben gibt es insgesamt keinen Grund, dass CED-Patienten ohne immunsuppressive Therapie und ohne weitere Komorbiditäten nicht an Arbeitsplätzen mit einer hohen Zahl an sozialen Kontakten mit Personen ohne besondere Risikokonstellation (z. B. Kunden im Supermarkt, Kinder in KITA und Schule o. Ä.) eingesetzt werden.

Medizinisches Personal hat überdurchschnittlich häufig Kontakt zu COVID-19-Patienten; andererseits besteht bei korrekter Anwendung der Personenschutzausrüstung kein erhöhtes Risiko für eine Infektion [38]. In der aktuellen pandemischen Situation wurden aus China und Italien [9, 59] Infektionsraten bei Krankenhausmitarbeitern von bis zu $10 \%$ bzw. $9 \%$ berichtet. Am 10.04.2020 wurden im täglichen Lagebericht des Robert Koch-Instituts (RKI) zur Coronavirus-Erkrankung unter den nach Infektionsschutzgesetz (IfSG) übermittelten COVID-19-Fällen in Deutschland 15495 Personen gezählt, die in medizinischen Einrichtungen gemäß § 23 Abs. 3 IfSG tätig sind. Zu diesen Einrichtungen zählen z. B. Krankenhäuser, Arztpraxen, Dialyseeinrichtungen, ambulante Pflegedienste und Rettungsdienste [60]. Die Zahl der Personen im Alter von 20 bis 64 Jahren in der Gesamtbevölkerung in Deutschland betrug 201848.950 .000 [61]. 2020 sind 45.130.000 Personen (92,2\%) erwerbstätig gewesen [62]. Davon arbeiten 5.700.000 im Gesundheitswesen. 116676 Personen im Alter von 20 bis 69 Jahren waren bis zum 27.04.2020 an einer SARS-Cov2-Infektion erkrankt [60]. Nimmt man hier einen Anteil von 92 \% Berufstätigen an, ergibt sich eine kalkulierte Zahl von ca. 107.000 Berufstätigen mit COVID-19-Erkrankung. Bei allen Unschärfen, die diese Abschätzung hat, ist somit der Anteil der gemeldet Erkrankten unter dem medizinischen Personal mit $0,27 \%$ numerisch höher als der Anteil bei den Gesamtberufstätigen $(0,23 \%)$ (siehe auch - Tab. 4). Ein Selektionsbias durch vermehrte Testungen medizinischen Personals auch schon bei milder Symptomatik kann dabei als Ursache der höheren Infektionsrate nicht ausgeschlossen werden

Passend dazu eine systematische Untersuchung aus dem Universitätsklinikum Münster bei insgesamt 957 Mitarbeitern insgesamt 52 Mitarbeiter (5,4\%) mit positiven SARS-CoV-2-Abstrichbefunden aus. Davon waren 33 Personen in einem Bereich mit direktem Patientenkontakt eingesetzt. Nach Einführung der Pflicht, einen Mund-Nasen-Schutz zu tragen, konnte in der infektionsepidemiologischen Nachverfolgung keine nosokomiale Person-zu-Person(Mitarbeiter/Patienten)-Übertragung mehr festgestellt werden [57]. 
In diesem Zusammenhang ist auch eine Fallkontrollstudie der GETAID mit 482 CED-Patienten, die im Gesundheitssystem arbeiten, von Interesse. Bei einer medianen Nachbeobachtung von 9,3 Jahren traten insgesamt 139 Infektionen (30 CDI, 33 schwere Virusinfektionen, 9 TBC-Fälle, 21 Pneumonien und 46 andere Infektionen) auf. Als Risikofaktoren konnten Steroide (OR: 3,05; $95 \%$ KI: 2,06-4,52), TNF-Antikörper (OR: 2,93; $95 \%$ KI: 2,024,27 ) und Immunsuppressiva (OR: 1,98; 95 \%-KI: 1,38-2,84) identifiziert werden. Nach dieser Studie haben CED-Patienten, die im Gesundheitssystem arbeiten, generell kein erhöhtes Risiko für Infektionen; Patienten mit einer immunsuppressiven Therapie sind aber besonders gefährdet. Somit erscheint eine Beschränkung der Arbeitsmöglichkeiten für CED-Patienten mit immunsuppressiver Therapie in Bereichen mit einem besonders hohen Infektionsrisiko (wie z. B. COVID-19-Stationen) gerechtfertigt.

In dem am 16. April 2020 vorgestellten SARS-CoV-2-Arbeitsschutzstandard des Bundesministeriums für Arbeit und Soziales [63] ist geregelt, dass Erwerbstätige, die Risikogruppen angehören, besonders geschützt werden müssen. Dazu sollten sich Beschäftigte individuell vom Betriebsarzt auch zu besonderen Gefährdungen wie einer möglichen COVID-19-Erkrankung beraten lassen. Vor dem Hintergrund des aktuellen Arbeitsplatzes hat der Betriebsarzt dem Arbeitgeber geeignete Schutzmaßnahmen vorzuschlagen, wenn die normalen Arbeitsschutzmaßnahmen nicht ausreichen. Gegebenenfalls kann der betroffenen Person auch ein Tätigkeitswechsel empfohlen werden. Dabei verpflichtet das Arbeitsschutzgesetz den Arbeitgeber dazu, die Gefahren für die Sicherheit und Gesundheit seiner Beschäftigten am Arbeitsplatz zu beurteilen (sogenannte Gefährdungsbeurteilung) und daraus Maßnahmen abzuleiten; diese sind ggf. im Rahmen der Pandemieplanung zu erweitern. So kann z. B. ein Tätigkeitswechsel für die Dauer der Pandemie sinnvoll sein.

\section{Kapitel 2: Diagnostik}

\section{EMPFEHLUNG 2.1}

Während der Pandemie sollten Patientenvorstellungen in Einrichtungen des Gesundheitswesens restriktiv erfolgen. CEDSprechstunden sollten unter Berücksichtigung der Dringlichkeit der Vorstellung und unter Optimierung der Infektionsschutzmaßnahmen wie räumlicher Distanzierung und nach Ausnutzen von Möglichkeiten der Telemedizin fortgeführt werden.

Empfehlungsgrad: EL5/Zustimmungsgrad: Konsens (86\%)

CED unterliegen einer Schwankung der Entzündungsaktivität und damit verbundenen Komplikationsmöglichkeiten und bedürfen je nach Schweregrad und Therapieeinstellung einer regelmäßigen Kontrolle [64]. Patienten mit akuten Beschwerden müssen weiterhin einen ungehinderten Zugang zu adäquater medizinischer Versorgung haben. Im Speziellen weisen CED-Patienten teils den Bedarf der unverzüglich erforderlichen Diagnostik und Therapie auf, wie z. B. beim Intraanalabszess, Ileus oder Zeichen eines fulminanten Verlaufes [65]. Daher kann generell auf die Versorgung in fachspezifischen Praxen und Zentren nicht verzichtet werden. Zu beachten ist auch, dass Restriktionen im Zugang zu Einrichtungen des Gesundheitssystems nicht zu einer Unterversorgung von CED-Patienten ohne SARS-CoV-2-Infektion führen dürfen.

Die hohe Rate von immunsuppressiven Therapien bei Patienten mit CED und die damit möglicherweise verbundene höhere Anfälligkeit für virale Infektionen [47] müssen zu einer erhöhten Vigilanz im Umgang mit Infektionsschutzmaßnahmen führen. Die Erfahrungen aus Regionen und Ländern mit einer großen Zahl an COVID-19-Patienten zeigen, dass das Risiko der Übertragung in medizinischen Einrichtungen in Zeiten der Pandemie hoch sein kann $[9,59,66]$.

Zum Schutz der Patienten, aber auch zum Schutz des „Gesundheitswesens“ sollte versucht werden, die Zahl der Besuche von Patienten in Einrichtungen des Gesundheitswesens möglichst gering zu halten [67]. Auf Routinevorstellungen von CED-Patienten in Remission sollte möglichst verzichtet werden. Eine persönliche Vorstellung von Patienten orientiert sich daher an der medizinischen Dringlichkeit, die der behandelnde Arzt mit dem Patienten individuell festlegen muss. Das Nutzen-Risiko-Verhältnis ist bei Kindern und Jugendlichen anders als bei Erwachsenen, und der Verzicht auf persönliche Vorstellungen ist mit einem größeren Risiko für eine unzureichende Behandlung der Grunderkrankung behaftet. Pädiatrische Patienten haben einerseits einen aggressiveren Krankheitsverlauf und in der Pubertät eine schlechte Therapieadhärenz und andererseits ein geringeres SARS-CoV-2-Infektionsrisiko in einer Kinderklinik im Vergleich zur Erwachsenenklink. Die verminderte Zahl an Ambulanzbesuchen erfordert immer alternative Kontaktaufnahmen, um sich über den Erkrankungszustand der Patienten mit CED zu informieren und die Behandlungsqualität nicht zu gefährden. Alternativ zu einer persönlichen Vorstellung, insbesondere bei Patienten in Remission, sollten die Möglichkeiten von Telefon- oder Videosprechstunden vermehrt genutzt werden. Die zunehmenden Möglichkeiten der Telemedizin im Bereich CED können dabei vermehrt zum Einsatz kommen [68, 69].

Bei persönlichen Visiten sei auf die allgemein bekannten Desinfektionsmaßnahmen, Abstandsregelungen, das Tragen von Mund-Nasen-Schutz zum Fremdschutz, aber auch zum Eigenschutz hingewiesen [70]. Weiterhin sollten Patienten grundsätzlich über Infektionsschutzmaßnahmen informiert werden und, wie in allen Hausarztpraxen üblich, vor Betreten der Räumlichkeiten telefonischen Kontakt aufnehmen, wenn Infektionszeichen bestehen oder ein Kontakt zu positiv Getesteten bestanden hat [71].

Räumliche Veränderungen wie Abstände innerhalb von Wartebereichen und die Distanzierung der Wartezone vom Behandlungsbereich (Parkplatz, Auto, vorgelagerte Flure, Treppenhaus etc.), aber auch die Optimierung der Patientenströme durch Terminverlässlichkeit und Verhinderung von unnötigen Ansammlungen durch Ausschließen von Angehörigen, Zusenden von Rezepten und Formularen können einen Beitrag zur Infektionsprävention leisten.

Auf elektive Endoskopien sollte bei CED-Patienten im Rahmen der Pandemie zum Schutz sowohl der Patienten als auch des medizinischen Personals möglichst verzichtet werden. Die Indikationsstellung zur Endoskopie muss im Rahmen einer individuellen Risiko-Nutzen-Abwägung erfolgen. Regional unterschiedliche In- 
- Tab. 5 Risikostratifizierung modifiziert nach den Empfehlungen der ESGE [87].

Patienten mit geringem Risiko

Patienten mit hohem Risiko
- keine Symptome

- kein wissentlicher Kontakt zu COVID-19-Patienten innerhalb der letzten 14 Tage

- keine Reisen in Gebiete/Regionen mit sehr hohen Raten an COVID-19-Neuerkrankungen innerhalb der letzten 14 Tage

Vorliegen von Symptomen, aber

- kein wissentlicher Kontakt mit COVID-19-Patienten

- keine Reisen in Hochrisikogebiete innerhalb der letzten 14 Tage

keine Symptome, aber

- Kontakt mit COVID-19-Patienten

- Aufenthalt in Regionen oder Umfeld mit hoher Infektionsrate innerhalb der letzten 14 Tage wenigstens ein Symptom + zusätzlich

- Kontakt mit COVID-19-Patienten

- Aufenthalt in Regionen oder Umfeld mit hoher Infektionsrate innerhalb der letzten 14 Tage fektionslagen sollten dabei Berücksichtigung finden. Alternativ zu einer invasiven Diagnostik könnten zur Überprüfung der Krankheitsaktivität z. B. die Durchführung von Calprotectin-Bestimmungen im Stuhl [72, 73], eine Ultraschalluntersuchung oder ggf. auch MRT-Untersuchungen genutzt werden. Möglichkeiten zur Calprotectin-Selbstmessung stehen als „Home-testing“-System bereits zur Verfügung [74]. Der Darm-Ultraschall hat sich sowohl bei MC als auch bei der CU zur Bestimmung der Krankheitsaktivität und zur Evaluation von Komplikationen, aber auch zum Krankheitsmonitoring als sehr hilfreich erwiesen [75, 76]. Das Infektionsrisiko bei nichtinvasiven Darm-Ultraschalluntersuchungen oder bei MRT-Untersuchungen ist sicherlich geringer als bei endoskopischen Untersuchungen. Dennoch müssen auch dabei die notwendigen Hygienemaßnahmen beachtet werden.

Alle während der Pandemie eingeleiteten restriktiven Maßnahmen, die den Patientenkontakt einschränken, sollten kontinuierlich mit den Zielen der Behandlungsqualität abgeglichen werden. Falls ambulante oder stationäre Vorstellungen in gesundheitlichen Einrichtungen als notwendig erachtet werden, sollten die üblichen Schutzmaßnahmen (z. B. Mund-Nasen-Schutz bei Patienten und Untersuchern) eingehalten werden.

\section{EMPFEHLUNG 2.2}

Während der Pandemie sollten sämtliche endoskopischen Untersuchungen unter besonderen Schutzmaßnahmen stattfinden. Das Ausmaß der Schutzmaßnahmen sollte risikoadaptiert bestimmt werden.

Empfehlungsgrad: EL5/ Zustimmungsgrad: starker Konsens (98\%)

Das Endoskopiepersonal ist über eine mögliche Inhalation von Tröpfchen und Aerosolen, Kontakt über die Konjunktiven sowie über den Stuhl einem erhöhten Risiko einer Infektion mit SARSCoV-2 ausgesetzt. Eine vermehrte Transmission der Infektion in Krankenhäusern durch infizierte Mitarbeiter wurde beschrieben [77, 78]. Endoskopien stellen einen Ort mit besonders hohem Übertragungsrisiko bakterieller und viraler Infektionen dar, sodass die Mitarbeiter dort in besonderer Weise geschützt werden sollten [79]. Während der Endoskopien kommt es zur Aerosolbildung.
Aerosole wurden als potenzielle Transmitter von SARS-CoV-2 beschrieben [80]. Darüber hinaus kommt es im Rahmen der COVID-19-Erkrankung bei den meisten Patienten zu einer Ausscheidung von Virus-RNA im Stuhl [37, 81-83]. Der Rezeptor für SARS-CoV-2, der ACE2-Rezeptor, ist im Gastrointestinaltrakt in hoher Zahl exprimiert, insbesondere im Bereich des terminalen lleums, in etwas geringerem Ausmaß auch im Kolon [84]. Die Ausscheidung konnte in einer Studie bis zu 21 Tage nachgewiesen werden [37]. Eine Transmission von SARS-CoV-2 über Aerosole, die im Rahmen von Koloskopien auftreten, ist daher nicht auszuschließen, wenngleich bisher kein infektiöses Virus aus dem Stuhl kultiviert werden konnte [37].

Infektionspräventive Maßnahmen haben sich insbesondere im Bereich der Endoskopie als hochgradig effektiv erwiesen [85, 86]. Bedauerlicherweise müssen wir bei allen vorgeschlagenen bzw. notwendigen Maßnahmen beachten, dass es zum Teil limitierte Ressourcen an Schutzausrüstung gibt und die Umsetzung der Empfehlungen daher leider nicht flächendeckend und zu jedem Zeitpunkt gewährleistet werden kann. Zwischenzeitlich wurden verschiedene Leitlinien für Verhaltensmaßnahmen bei Endoskopien in Zeiten der SARS-CoV-2-Pandemie verfasst, u. a. von der ESGE [87] (siehe $>$ Tab. 5), der AGA [88] und der Asian Pacific Society for Digestive Endoscopy[89]. Eine Risikostratifizierung und ein risikoadaptiertes Vorgehen in Analogie zu den ESGE-Leitlinien erscheinen sinnvoll und pragmatisch [87]. Derzeit variiert die Infektions- und Gefährdungslage in Deutschland von Region zu Region zum Teil erheblich. Zu den Hochrisikogruppen zählen in jedem Fall Personen mit oder ohne Symptome, die innerhalb der letzten 14 Tage Kontakt zu COVID-19-Patienten hatten. Personen, die sich innerhalb der letzten 14 Tage in Regionen mit derzeit starker Verbreitung von SARS-CoV-19-Infektionen oder in einem Umfeld mit einer Ausbruchssituation aufgehalten haben (z. B. in Altenheimen oder Krankenhäusern), zählen ebenfalls zu einer Hochrisikogruppe. Die AGA fordert flächendeckend Schutzmaßnahmen wie bei SARS-CoV-2-positiven Patienten, da im Rahmen einer Pandemie das Risiko überall als gleich hoch einzustufen sei [88]. Dieser Empfehlung können sich die Konsensusteilnehmer in der Mehrheit nicht anschließen, da sich diese Situation nicht mit der in Deutschland regional sehr unterschiedlich vorliegenden Prävalenz der Erkrankung deckt. Ein risikoadaptiertes Vorgehen 
erscheint daher den meisten Konsensusteilnehmern auch unter Berücksichtigung der zeitweilig limitierten Schutzausrüstung als sinnvoll und ausreichend.

Allgemeine Maßnahmen, die bei allen Endoskopien berücksichtigt werden sollten, beinhalten, dass in Zeiten der Pandemie eine enge Indikationsstellung erfolgen sollte. Der Schutz des Personals hat oberste Priorität. Bei jeder endoskopischen Untersuchung sollte geprüft werden, ob es keine alternativen diagnostischen Verfahren gibt. Das Endoskopiepersonal sollte in kleinen Teams möglichst getrennt arbeiten, gemeinsame Treffen bzw. Veranstaltungen innerhalb des Teams sollten vermieden werden. Eine professionelle Hygieneschulung sollte erfolgen, und die eingeleiteten Schutzmaßnahmen sollten von Hygienefachkräften begleitet werden. Die Hygieneschulung sollte u. a. allgemeine Hygienemaßnahmen, Risikofaktoren für COVID-19 und die korrekte An- und Ablage der Schutzkleidungen umfassen. Grundsätzlich sollte Einmalmaterial verwendet werden. Die Endoskopieaufbereitung erfolgt nach den allgemeinen Regeln. Nach jeder endoskopischen Untersuchung erfolgt eine postinterventionelle Flächendesinfektion mit viruziden Substanzen [87]. Generell sollte berücksichtigt werden, dass die erforderlichen Hygienemaßnahmen in der Endoskopie incl. der Verwendung der Schutzausrüstung auch von der aktuellen und regionalen Infektionslage abhängen und nach Rücksprache mit den Hygieneexperten vor Ort auch modifiziert werden können.

Das Risikomanagement vor der Endoskopie umfasst eine Risikostratifizierung, die am Tag der Untersuchung wiederholt werden sollte. Endoskopie- und Aufwachbereiche sollten gemäß der Hoch- und Niedrigrisiko-Stratifizierung getrennt werden. Alle Patienten, die die Endoskopie betreten, sollten Mund-Nasen-Schutz tragen [87]. Eine generelle Testung der Patienten (z. B. Nasen-Rachen-Abstrich) vor Eintritt in die endoskopische Einheit wird von der Mehrheit der Konsensuskonferenzteilnehmer als nicht notwendig angesehen. Personen, die symptomatisch bzgl. einer COVID-19-Infektion sind und die zur Hochrisikogruppe zählen, sollten ggf. vor einer endoskopischen Untersuchung getestet werden. Die Kontrolle der Körpertemperatur des Patienten vor Betreten der Behandlungseinheit, ggf. auch das Ausfüllen eines strukturierten Risikoerfassungsfragebogens, wie er von vielen Kassenärztlichen Vereinigungen angeboten wird, kann sinnvoll sein und wird vom RKI empfohlen.

Das Risikomanagement während der Endoskopie umfasst den Einsatz der persönlichen Schutzausrüstung bei allen endoskopischen Untersuchungen. In Abhängigkeit vom Risikostatus sollte unterschiedliche Schutzausrüstung verwendet werden (siehe - Tab.6). Patienten, die auf der Intensivstation liegen, sollten direkt auf dieser Behandlungseinheit endoskopiert werden.

Bezüglich der von der ESGE formulierten Empfehlung zur Durchführung von Endoskopien bei Hochrisikopatienten in „Endoskopie-Räumen mit Unterdruck“ konnte bei den Konsensusteilnehmern keine einheitliche Zustimmung erreicht werden, zumal die Verfügbarkeit dieser Räume limitiert ist. Das Arbeiten in gut ventilierten Räumen erschien den meisten Teilnehmern ausreichend zu sein bzw. eine pragmatische Alternative darzustellen. Alternativ können COVID-19-Patienten auch in Operationssälen (mit Unterdruck) endoskopiert werden.
D Tab. 6 Risikoabhängige Schutzausrüstung in der Endoskopie [87].

\begin{tabular}{l|l|}
$\begin{array}{l}\text { Patienten mit } \\
\text { geringem Risiko }\end{array}$ & $\begin{array}{l}\text { Mund-Nasen-Schutz } \\
\text { Handschuhe } \\
\text { Schutzbrille oder Schutzschild } \\
\text { wasserfester langärmeliger Einmalkittel }\end{array}$ \\
\hline $\begin{array}{l}\text { Patienten mit } \\
\text { hohem Risiko } \\
\text { Gesicherte } \\
\text { COVID-19-Infektion }\end{array}$ & $\begin{array}{l}\text { FFP2- oder FFP3-Maske } \\
\text { doppelte Handschuhe } \\
\text { Haarschutz } \\
\text { Schutzbrille oder Schutzschild } \\
\text { wasserfester langärmeliger Einmalkittel }\end{array}$ \\
\hline
\end{tabular}

Einige Fachgesellschaften bzw. Autoren haben sich bereits mit der Frage beschäftigt, wie es nach der Pandemie mit endoskopischen Untersuchungen weitergeht [90-92]. In der Regel wird eine risikoadaptierte, stufenweise Rückkehr zum Normalbetrieb („neue Normalität“) favorisiert. Erfahrungen aus der schrittweisen Öffnung der Endoskopien in China und anderen besonders betroffenen Regionen sind sicher hilfreich.

Innerhalb des Behandlungsteams sollte eine gegenseitige Kontrolle bzgl. der Einhaltung von Hygiene- und Schutzregeln erfolgen. Auf eine Verminderung der Aerosolbildung sollte geachtet werden. Dies kann einerseits durch vorsichtiges Entfernen von Zubehör aus dem Arbeitskanal erreicht werden, aber auch durch eine kontinuierliche Absaugung während der Entfernung von Zubehör aus dem Arbeitskanal. Die kontinuierliche Absaugung am Endoskop bei der Entfernung von Zubehör wie Zangen, Schlingen etc. reduziert die Aerosolbildung erheblich und sollte daher grundsätzlich im Rahmen von Endoskopien erfolgen [93].

Da sich die Erkenntnisse bezüglich des Erkrankungs- und Übertragungsrisikos bei COVID-19-Erkrankung im ständigen Wandel befinden, ist auch bezüglich der endoskopischen Untersuchungen zu erwarten, dass die entsprechenden Empfehlungen neuen Erkenntnissen angepasst werden müssen.

\section{Antikörperdiagnostik}

\section{EMPFEHLUNG 2.3}

Eine SARS-CoV-2-Antikörpertestung kann ein besseres Patientenmanagement ermöglichen. Die Testung birgt zum jetzigen Zeitpunkt jedoch noch Probleme in der Verfügbarkeit und im sicheren Nachweis einer Immunität.

Empfehlungsgrad: EL5/Zustimmungsgrad: Konsens (86\%)

Prinzipiell wäre das Wissen um die Immunantwort bei der CEDPatientenversorgung von großem Interesse: Immunsuppressive Therapien können möglicherweise die Empfänglichkeit für eine virale Infektion erhöhen (siehe Empfehlung 1.2), ein unkontrollierter Entzündungsstatus den Verlauf der SARS-CoV-2 beeinflussen [94]. Das Wissen um eine Immunität der Patienten könnte sowohl den Umgang in den behandelnden Abteilungen als auch die Wahl der Medikation einer Neueinstellung und generelle Verhaltensempfehlungen, etwa bzgl. der Berufsausübung in publikumsreichen Berufen, beeinflussen. 
Die momentan in Deutschland angewandte IgA-Testung von Euroimmun lässt nur eingeschränkt einen akuten Infektionsnachweis zu und ist der PCR-Testung in der Akuttestung deutlich unterlegen. Als Verlaufstestung wird dieser Test angeboten, um die beginnende Immunität nachzuweisen [95]. Dazu ist eine Spezifität von $90 \%$ allerdings nicht ausreichend. Kreuzreaktionen mit anderen Coronaviren sind zu häufig. Daher ist dieser Test zum Screening nicht geeignet.

Die IgG-Testung lässt eine Spätphase der Infektion und Beschreibung der Immunität zu. Durch die hochspezifische S1-Domäne des Spike-Proteins erreicht der Test eine hohe Spezifität von $99 \%$ [96]. Weiterhin stellt sich die Frage nach dem Zeitpunkt der Testung; wahrscheinlich ist ein multiples Testen (z. B. bei jeder Kontrollblutentnahme) notwendig, um auch inapparente Verläufe zu sichern [97].

\section{Kapitel 3: Therapieanpassung}

Zum jetzigen Zeitpunkt ist nicht vollständig geklärt, welche Konsequenzen sich für die medikamentöse und operative Therapie bei Patienten mit CED aus der aktuellen Risikokonstellation einer Corona-Pandemie ergeben. Die Evidenz von Empfehlungen stützt sich zurzeit im Wesentlichen auf Erfahrungen bei anderen Viruserkrankungen, etwa einer früheren SARS-Epidemie, MERS-Epidemie, und die Erfahrungen mit Influenzaerkrankungen sowie auf Erkenntnisse aus der aktuellen Corona-Pandemie in China und einem internationalen Register der IOIBD (Secure-IBD) (siehe unten) sowie daraus abgeleitete Expertenempfehlungen der IOIBD [54]; dieser Umstand sollte klar an Patienten kommuniziert werden. Zudem sind Sorgen und Ängste ernst zu nehmen, und es ist individuell zu entscheiden.

\subsection{Medikamentöse Therapie}

\section{EMPFEHLUNG 3.1}

Patienten mit einer CED und einer immunsuppressiven Therapie haben generell kein erhöhtes Risiko für einen schweren Verlauf einer SARS-CoV-2-Infektion. Eine immunsuppressive Therapie sollte deshalb bei einer leichten bis moderaten COVID-19-Erkrankung nicht reduziert werden. Ausnahmen bilden die längerdauernde Therapie mit systemischen Steroiden insbesondere in Dosierungen größer 20 mg Prednisonäquivalent/Tag. Diese sollte daher möglichst vermieden oder, soweit klinisch vertretbar, reduziert und beendet werden. Empfehlungsgrad: EL3/Zustimmungsgrad: Konsens (84\%)

Ohne Zweifel ist insgesamt das Risiko für Infektionen bei Patienten mit einer immunsuppressiven Therapie bzw. einer Steroidmedikation signifikant erhöht; diese bedingen auch eine erhöhte Mortalität [39]. Im Gegensatz dazu scheint die Behandlung z. B. mit Vedolizumab das Risiko für Infektionen im Respirationstrakt nicht zu erhöhen [52]. Derzeit ist unklar, welche Konsequenzen sich aus dieser Risikokonstellation ergeben. Evidenzbasierte Empfehlungen, die sich aus kontrollierten Studien bzw. Interventions- studien ergeben, können nicht gegeben werden; dieser Umstand ist klar an Patienten zu kommunizieren. Deren Sorgen und Ängste sind ernst zu nehmen und gemeinsam individuelle Entscheidungen im Sinne des „shared decision making“ zu treffen: Was spricht für ein Aussetzen, was spricht dagegen? Ein Aussetzen der immunsuppressiven Therapie oder eine Reduktion der Dosis bergen das Risiko, dass die Grunderkrankung sich verschlechtert. So wird in der ersten am 13.03.2020 veröffentlichten Einschätzung der ECCO betont, dass ein Absetzen oder eine Dosisreduktion zur Risikoreduktion für eine COVID-19-Erkrankung nicht empfohlen werden [98]. Es ist somit fraglich, ob eine Verlängerung der Infusionsintervalle bei Patienten unter einer Biologikatherapie in stabiler Remission diesen wirklich nützt. Es gibt lediglich Daten zur Möglichkeit der Verlängerung des Infliximab-Infusionsintervalls auf 10 Wochen bei Patienten in stabiler Remission [99]. Die Sinnhaftigkeit dieser Maßnahmen liegt, wenn überhaupt, darin, Kontakte zu Einrichtungen des Gesundheitssystems zu minimieren (siehe dazu auch Empfehlung 3.7). So zeigen kritische Aufarbeitungen aus China, dass bis zu $41 \%$ der COVID-19-Erkrankungen als nosokomiale Infektionen verstanden werden müssen [100].

Klarer ist die Situation für Empfehlungen, die eine Steroidtherapie betreffen. Die verfügbaren Daten zu diesen Konzepten weisen auf eine erhöhte Mortalität und sekundäre Infektionen bei Influenzapatienten hin, die eine Steroidtherapie erhalten. So wurde eine verzögerte Elimination von SARS-CoV und MERS-CoV bei Überlebenden mit Steroiden beschrieben [101]. Auf der anderen Seite sind aber bei Patienten mit COVID-19-assoziiertem ARDS (Acute Respiratory Distress Syndrome) Steroide protektiv [102]. Im IO IBD-Register haben jedoch Patienten mit einer systemischen Steroidtherapie die höchste Mortalität im Vergleich zu Patienten, die mit anderen Substanzen behandelt wurden (siehe dazu auch $>$ Abb.4). Systemisch wirkende Steroidtherapien, insbesondere in Dosierungen über $20 \mathrm{mg} /$ Tag, sollten somit vermieden werden.

Divergente Einschätzungen ergeben sich zu Medikamenten, die eine Lymphozytopenie bewirken (Azathioprin, 6-Mercaptopurin) bzw. die Signaltransduktion in Lymphozyten hemmen (Calcineurininhibitoren, JAK-Inhibitoren). Eine niedrige Zahl von CD4 ${ }^{+}$-T-Zellen ist mit verzögerter SARS-CoV-2-Viruselimination und einem schweren Verlauf assoziiert [103], sodass bei manifester und schwerer Erkrankung ein Stopp der Therapie mit Thiopurinen oder JAK-Inhibitoren begründet sein mag [104]. Die Auswertung der Patientenverläufe von Patienten mit CED und gesicherter SARS-CoV-2-Infektion aus dem IO IBD-Registern zeigt, dass die Komplikationsrate (Aufnahme auf die Intensivstation/Notwendigkeit zur Beatmung/Tod) von der Art der Behandlung der Grunderkrankung abhängt. In diesem Register wurden bis zum 23.06.2020 über 1500 Patienten dokumentiert, sodass eine relevante Datenlage vorliegt.

EMPFEHLUNG 3.2

Bei Patienten mit schwerer COVID-19-Erkrankung sollte die Therapie mit Thiopurinen, Methotrexat und Tofacitinib pausiert und nach Überwinden der Infektion wieder aufgenommen werden.

Empfehlungsgrad: EL5/Zustimmungsgrad: Konsens (94\%) 


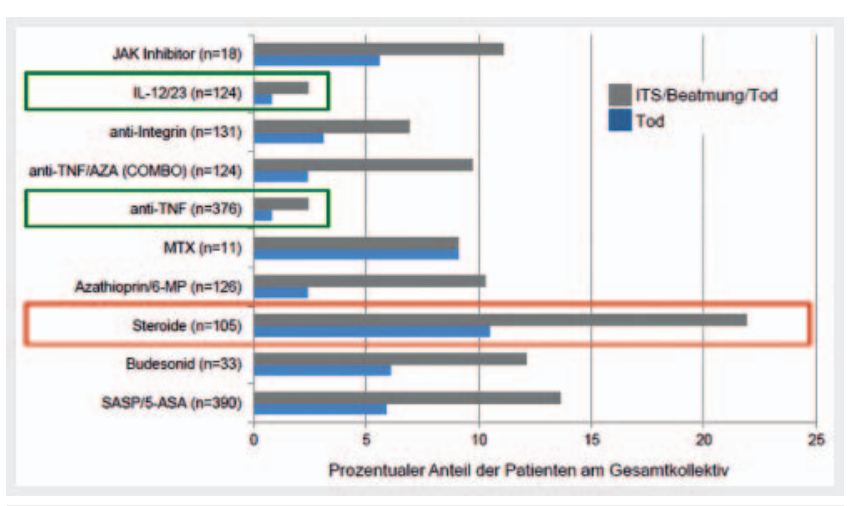

- Abb. 4 Schwere COVID-19-Erkrankungsverläufe bei CED-Patienten unter verschiedenen Therapeutika (Secure-IBD-Register der IOIBD, letzte Abfrage: 26.05.2020). Dargestellt ist der prozentuale Anteil der Patienten, die verstorben sind bzw. den kombinierten Endpunkt (Aufenthalt auf einer ITS-Station/Beatmungspflicht/Tod) erlitten haben, in Abhängigkeit von der medikamentösen Behandlung. Insgesamt gingen in die Auswertung die Daten von $1302 \mathrm{~Pa}-$ tienten ein; dabei ergibt die Summe der zu den einzelnen Medikamenten aufgelisteten Patienten einen höheren Wert, da auch Kombinationstherapien durchgeführt wurden. Auffällig ist die hohe Sterblichkeitsrate bei Patienten, die mit Steroiden (oral/parenteral) behandelt wurden (rotes Rechteck), im Vergleich zu günstigen Verläufen bei Patienten, die mit TNF-Antikörpern bzw. IL12 / IL23-Antikörpern behandelt wurden. Die Gruppe der Patienten, die einen JAK-Inhibitor erhielten, ist mit $n=18$ zu klein, um belastbare Aussagen zum Verlauf abzuleiten.

Bei Medikamenten, die eine Lymphozytopenie bewirken können (Azathioprin, 6-Mercaptopurin, Tofacitinib, Methotrexat), soll im Fall einer SARS-CoV-2-Infektion insbesondere bei schwerer Symptomatik eine Beendigung oder Pausierung der Therapie erwogen werden, wenngleich die lange Halbwertszeit von Thiopurinen und MTX dieser Maßnahme möglicherweise widerspricht. Eine niedrige Zahl an CD4+-T-Zellen ist jedoch mit verzögerter SARS-CoV-2Viruselimination und einem schweren Verlauf assoziiert [103]. In einer großen Fallserie mit mehr als 15000 Patientenjahren (PJ) (4800 PJ mit Thiopurinen, 3000 PJ mit Anti-TNF-Therapie) zeigten sich 31 Fälle schwerer viraler Infektionen (EBV, CMV, VZV und HZV), davon die meisten bei Patienten mit einer Thiopurintherapie [45], sodass bei manifester COVID-19-Erkrankung ein Stopp der Therapie mit Thiopurinen, Tofacitinib und Methotrexat begründet sein mag [33, 104]. Ein erneuter Therapiebeginn wäre nach 2 negativen SARS-CoV-2-PCR-Tests aus dem Nasen-Rachen-Abstrich und Besserung der klinischen Symptomatik der COVID-19-Erkrankung möglich. Für MTX liegen keine spezifischen Daten für eine SARS-CoV-2Infektion vor. Ein erneuter Therapiebeginn wäre nach 2 negativen SARS-CoV-2-PCR-Tests aus dem Rachenabstrich möglich.

Tofacitinib stellt als JAK-1/-3-Inhibitor im Gegensatz zu JAK-2Inhibitoren, die möglicherweise den Viruseintritt hemmen und den Th17-vermittelten Zytokinsturm dämpfen [105], keinen unmittelbaren therapeutischen Ansatz zur Behandlung des Zytokinsturms bei der COVID-19-Erkrankung dar. Die Therapie mit Tofacitinib geht mit gehäuften viralen Infektionen (VZV) einher [106]. In-vitro-Studien zeigen, dass Tofacitinib die Produktion von Interferon- $\boldsymbol{\alpha}$ hemmt [107], ein Phänomen, das in der ersten Phase der Immunantwort eher ungünstig ist [108]. Aufgrund der kurzen Halbwertszeit sind deshalb ein Absetzen und ggf. der Wiederbeginn nach Ausheilung der COVID-19-Erkrankung sowie nach 2 negativen SARS-CoV-2-PCR-Untersuchungen sinnvoll. Wie oben schon ausgeführt, werden insbesondere JAK-2-Inhibitoren zur Therapie des Hyperinflammationsyndroms bei schwerer COVIDErkrankung eingesetzt [109, 110]; diese Konzepte sind aber von der Wirkung von Tofacitinib abzugrenzen.

\section{EMPFEHLUNG 3.3}

Bei Patienten in stabiler Remission unter kombinierter AntiTNF-Antikörper-/immunsuppressiver Therapie (Thiopurin, MTX) sollte das Immunsuppressivum abgesetzt werden. Empfehlungsgrad: EL5/Zustimmungsgrad: mehrheitliche Zustimmung: (73\%)

Wie bereits in Leitlinien empfohlen [111], sollte bei Erreichen einer stabilen Remission über mindestens sechs Monate [112] (klinische Remission CDAI < 150 bei Morbus Crohn oder Mayo Score $<3$ bei Colitis ulcerosa mit endoskopischem Subscore 0 oder 1, kein rektaler Blutabgang, fäkales Calprotectin $<250 \mu \mathrm{g} / \mathrm{g}$ Stuhl, zusätzlich CRP normwertig) unter einer kombinierten Therapie mit Azathioprin und Infliximab Azathioprin abgesetzt werden. In den der Cochrane-Metaanalyse [113] zugrunde liegenden Studien $[112,114]$ waren die Rückfallraten nach Umstellung auf eine Infliximab-Monotherapie nicht unterschiedlich (48\% Kombinationstherapie vs. 49\% Monotherapie, RR: 1,02; $95 \%-K I: 0,68-$ 1,52). Dennoch konnte für diese Empfehlung nur eine mehrheitliche Zustimmung mit $76 \%$ erreicht werden. Der positive Effekt von Azathioprin auf die Bildung von Autoantikörpern gegen Infliximab, individuelle Besonderheiten in der Vorgeschichte des Patienten (z. B. ein schwerer Verlauf, der nur durch eine COMBOTherapie in eine Remission überführt werden konnte), wurden als Argumente gegen diese Empfehlung genannt.

\section{EMPFEHLUNG 3.4}

Während der SARS-CoV-2-Pandemie sollte eine Biologikatherapie mit zu erwartendem raschem Wirkeintritt gegenüber einer hochdosierten systemischen Steroidtherapie im akuten Schub bevorzugt eingesetzt werden.

Empfehlungsgrad: EL5/Zustimmungsgrad: Konsens (85\%)

Um die möglicherweise risikoreiche hochdosierte systemische Steroidtherapie zu umgehen, bietet sich der Einsatz eines schnell wirksamen Biologikums an. Die Analyse von TNF-Spiegeln bei COVID-19-Patienten zeigte unterschiedliche Ergebnisse: In einer Studie wurden keine erhöhten TNF-Spiegel bei schweren Verläufen gemessen, obwohl andere proinflammatorische Zytokine erhöht waren [115]. In einer weiteren Studie zeigte sich hingegen, dass COVID-19-Patienten auf der Intensivstation höhere TNFSerumspiegel aufweisen als solche, die auf einer Normalstation behandelt werden konnten. TNF könnte pathogene Effekte bei COVID-19 potenzieren, da es die ACE2-Expression erhöht und 
eine Lymphozytopenie verstärken kann [116]. Diese Argumente einschließlich einer möglichen Suppression des Zytokinsturms und der rasche Wirkeintritt sprechen für einen Einsatz von AntiTNF-Medikamenten im Fall eines Schubs bei gleichzeitiger Vermeidung hochdosierter systemischer Steroide. Für Vedolizumab [117] und Ustekinumab konnte bei CED-Patienten keine erhöhte Rate viraler Infektionen beobachtet werden. Es wird spekuliert, dass die II-23-Blockade durch Ustekinumab bei COVID-19 die pathogene Th17-Antwort im Rahmen eines Zytokinsturms unterdrückt [94]. Wie oben angeführt, weist das IO IBD-Register für Patienten mit einer systemischen Steroidtherapie die höchste Mortalität im Vergleich zu Patienten aus, die mit Biologika behandelt werden.

\section{EMPFEHLUNG 3.5}

Eine Umstellung einer intravenösen Infliximab-Therapie auf ein subkutanes Therapiekonzept mit alternativen Biologika bei Patienten in Remission unter Infliximab sollte nicht erfolgen. Empfehlungsgrad: EL5/Zustimmungsgrad: Konsens (88\%)

\section{EMPFEHLUNG 3.6}

Bei Neueinstellungen einer Biologikatherapie sollte bei gleichwertiger Erfolgsaussicht eine s.c. Formulierung eingesetzt werden.

Empfehlungsgrad: EL5/Zustimmungsgrad: Konsens (80\%)
Es liegen nur begrenzte Daten vor, die eine sichere Verlängerung der Infusionsintervalle unterstützen. So ist eine Verlängerung des Infliximab-Infusionsintervalls auf 10 Wochen bei Patienten in stabiler Remission [99] möglich. Dies gilt nicht für Patienten mit verkürztem Infusionsintervall und/oder erhöhter Dosis pro Infusion. Eine stabile Remission sollte jedoch keinesfalls durch eine weitere Verlängerung der Infusionsintervalle bei SARS-CoV-2-negativen Patienten riskiert werden. Daten aus China und Südkorea bei pädiatrischen CED-Patienten belegen, dass bei Verlängerung des Intervalls zwischen Infliximab-Infusionen aus Sorge vor einer Infektion eine Exazerbation ihrer CED bei $20 \%$ der Patienten auftrat [30]. Möglicherweise ist bei einem Nachweis hochnormaler Infliximab-Talspiegel eine Verlängerung in Ausnahmefällen möglich; dafür wäre die Bestimmung von Talspiegeln vor den Infusionen sinnvoll, um keinen unerwünschten Wirkverlust zu provozieren. Die Steuerung der Therapie mit Vedolizumab mithilfe von Talspiegeln ist in der klinischen Praxis nicht etabliert. In stabiler Remission im 4-Wochen-Intervall können Vedolizumab-Infusionen auf längere Intervalle bis hin zu 8-Wochen-Intervallen ausgedehnt werden. Darüber hinaus wird durch die Marktzulassung ab Juni 2020 eine Umstellung auf eine s.c. Formulierung von Vedolizumab möglich sein [121].

\section{EMPFEHLUNG 3.8}

Bei Patienten mit nachgewiesener COVID-19-Erkrankung kann die Therapie mit TNF-Antikörpern, Ustekinumab und Vedolizumab im Einzelfall pausiert werden und nach Überwinden der Infektion wieder aufgenommen werden.

Empfehlungsgrad: EL5/Zustimmungsgrad: Konsens (86\%)

Die sicherste Therapievariante für SARS-CoV-2-negative Patienten unter einem intravenösen Therapieregime ist die Etablierung eines Non-COVID-19-Infusionsbereichs. In diesem Rahmen können Infusionen unter Berücksichtigung der dafür vorgeschriebenen Hygienemaßnahmen hinreichend sicher durchgeführt werden. Vor Betreten eines solchen Non-COVID-19-Infusionsbereichs ist sicherzustellen, dass bei den Patienten keine Krankheitssymptome vorliegen und ein chirurgischer Mund-NasenSchutz gestellt werden kann. Der Abstand zwischen den einzelnen Infusionsplätzen soll $2 \mathrm{~m}$ betragen. Die Wartezeit auf die Infusion soll minimal gehalten werden. Die Zubereitung der Infusion kann am Infusionstag nach telefonischem Kontakt mit Sicherstellung der Symptomfreiheit vorbereitet werden und dann rasch verabreicht werden. Eine Infusionsdauer von 60 Minuten bei Infliximab erscheint bei Erwachsenen und Kindern sicher [118-120]. Bis zur Zulassung einer s. c. Formulierung für IFX wäre eine Umstellung in der CED-Therapie ein Off-Label-Gebrauch, der nicht zu empfehlen ist.

\section{EMPFEHLUNG 3.7}

Eine Verlängerung der Infusionsintervalle für TNF-Antikörper oder Vedolizumab sollte nicht erfolgen.

Empfehlungsgrad: EL5/Zustimmungsgrad: Konsens (91\%)
Sollte die Gabe der o. g. Medikamente in die Phase einer schweren COVID-19-Erkrankung fallen, kann die Applikation um 14 Tage verschoben werden und nach Ausheilung der Erkrankung sowie nach 2 negativen SARS-CoV-2-PCR-Tests wieder aufgenommen werden. Diese Empfehlung trägt den bekanntermaßen teilweise langen Halbwertszeiten keine Rechnung. Die bisherigen Daten der Secure-IBD-Studie ergeben bisher auch keine Hinweise auf ein erhöhtes Risiko eines ungünstigen Verlaufs einer COVID-19Erkrankung (Intensivbehandlung, Mortalität) unter einer Therapie mit TNF-Antikörpern, Ustekinumab und Vedolizumab. TNF-Antikörper könnten sogar möglicherweise eine Verminderung des pulmonalen Organschadens bewirken [94]. Dieser Effekt wird vermutlich durch ein verringertes „Shedding“ der ACE2-Ektodomäne ausgelöst, das über TNF $\alpha$-Converting-Enzyme vermittelt wird. Die ACE2-Ektodomänen spielen eine wesentliche Rolle für das Eindringen von SARS-CoV-2 in die Zellen. Da hohe IFN- $\gamma$ - und TNF- $\alpha$-Spiegel mit schweren Verläufen einer SARS-CoV-Infektion assoziiert sind, wird eine Blockade von TNF als mögliche Option zur Behandlung dieses Zytokin-Release-Syndroms bei einigen Patienten postuliert [94]. 


\subsection{Chirurgische Therapie}

\section{EMPFEHLUNG 3.9}

Dringliche und notfallmäßige Operationen sollen in Zeiten der SARS-CoV-2-Pandemie unter adäquaten Schutzmaßnahmen durchgeführt werden.

Empfehlungsgrad: EL5/Zustimmungsgrad: starker Konsens (98\%)

In vielen Krankenhäusern sind Non-COVID-19-Operationssäle etabliert worden, sodass sich die zunächst vorgeschriebene Reduktion der Operationen auf lebensbedrohliche Situationen bereits deutlich entspannt. Die Indikation zur Operation sollte engmaschig in interdisziplinären Teams besprochen werden. Es sollte insbesondere dann eine Verschiebung der Operation kritisch evaluiert werden, wenn infolge der Verzögerung eine erhöhte Komplikationsrate droht.

\section{EMPFEHLUNG 3.10}

Bei Patienten mit isoliertem, kurzstreckigem Befall des terminalen Ileums bei Morbus Crohn kann eine primäre Resektion vs. eine Steroidstoßtherapie oder eine Biologikatherapie unabhängig von einer möglichen SARS-CoV-2-Infektion zur Remissionsinduktion erwogen werden.

Empfehlungsgrad: EL5/Zustimmungsgrad: Konsens (84\%)

Sollte bei isoliertem, kurzstreckigem Befall des terminalen lleums bei Morbus Crohn eine Budesonid-Therapie nicht ausreichend wirksam sein, ist bei Vorliegen obstruktiver Symptome eine vorübergehende ausschließliche enterale Ernährungstherapie zu erwägen (siehe Empfehlungen zur Ernährung). Bei Kindern und Jugendlichen ist sie in dieser Situation die erste Wahl [122]. Bei der ECCO 2020 wurde die Nachbeobachtungsstudie der Lir!c-Studie vorgestellt. Darin wurde nach 5 Jahren Beobachtungszeitraum ein Vorteil zugunsten einer Resektion des terminalen Ileums im Vergleich zu einer Anti-TNF-Therapie bei Patienten gesehen, die als Vortherapie lediglich Immunmodulatoren erhalten hatten [123]. Die bereits publizierten 4-Jahresdaten hatten diesen Unterschied zunächst nicht gezeigt [124].

\section{EMPFEHLUNG 3.11}

Bei Patienten mit akuter, schwerer, steroidrefraktärer Colitis ulcerosa soll in der SARS-CoV-1-Pandemie eine frühzeitige Proktokolektomie sorgfältig gegen eine Fortführung der medikamentösen Therapiestrategie abgewogen werden. Empfehlungsgrad EL5/Zustimmungsgrad: Konsens (78\%)

In dieser Situation sollte gemeinsam mit dem Patienten die Option einer Proktokolektomie gegen das Potenzial der in dieser
Situation grundsätzlich wirksamen Medikamente (Infliximab, Calcineurininhibitoren als Bridging-Therapie zu Vedolizumab) diskutiert und eine gemeinsame Entscheidung auch unter Berücksichtigung des Infektionsrisikos mit SARS-CoV-2 während des stationären Aufenthalts herbeigeführt werden.

\section{Kapitel 4: Adjuvante Therapie und besondere Fragestellungen}

\section{Impfung}

\section{EMPFEHLUNG 4.1}

Nicht immunisierte CED-Patienten sollten zum Schutz vor einer zusätzlichen Atemwegsinfektion gegen Influenza und Pneumokokken geimpft werden.

Empfehlungsgrad: EL 4 /Zustimmungsgrad: starker Konsens (97\%)

Die Impfung gegen Influenza und Pneumokokken schützt nicht vor SARS-CoV-2 selbst, minimiert aber die Gefahr von Doppelinfektionen. Daher ist ein vollständiger Impfschutz nach STIKOEmpfehlungen für alle, aber insbesondere für Risikopersonen wie Senioren und Personen mit schweren Vorerkrankungen wichtig [125]. Das gilt unabhängig von einer aktuellen oder geplanten immunmodulatorischen Therapie von CED-Patienten.

Die Impfung gegen Influenza schützt vor schwer verlaufenden Grippe-Infektionen, z. B. einer Pneumonie, die eine Versorgung der Patienten in einem Krankenhaus oder auf einer Intensivstation erforderlich machen. Die jährliche Impfung ist die wichtigste Präventionsmaßnahme gegen Influenza überhaupt und kann somit auch das Risiko für eine möglicherweise besonders schwer verlaufende Doppelinfektion mit dem Coronavirus reduzieren und die Wahrscheinlichkeit einer stationären Intensivbehandlung senken (RKI) [125].

Es gibt keine Hinweise darauf, dass die Auseinandersetzung des Immunsystems mit dem SARS-CoV-2 durch eine in zeitlicher Nähe verabreichte Impfung beeinflusst wird. Durch Impfungen wird der Impfling vor Infektionen geschützt, die ihn auch während der Pandemie zusätzlich gefährden oder schädigen können [125].

Das RKI empfiehlt, dass ausstehende Impfungen sofort durchgeführt werden, wenn ein Patient bereits in der Praxis ist. Insbesondere Patienten, die zu einer Risikogruppe für einen schweren Verlauf von COVID-19 gehören, sollten jedoch zurzeit nicht mit dem öffentlichen Personennahverkehr fahren und nicht in einem Wartezimmer mit weiteren Personen sitzen, um eine Impfung wahrzunehmen. Oberste Priorität hat im Moment die Kontaktreduzierung. Diese schützt nicht nur vor SARS-CoV-2-Infektionen, sondern auch vor der Übertragung anderer Infektionserkrankungen. Es kann daher individuell sinnvoll sein, Impfungen zu verschieben (Empfehlungen der Ständigen Impfkommission) [125]. 


\section{Studien}

\section{EMPFEHLUNG 4.2}

Während der SARS-CoV-2-Pandemie sollte der Einschluss in Interventionsstudien nach Alternativtherapien, Schweregrad der Erkrankung und potenzieller Schwächung der viralen Abwehr geprüft werden. In Studien eingeschlossene Patienten können weitergeführt werden. In Einzelfällen kann bei Risikopatienten und gemäß Wirkmechanismus der Studienmedikation mit Einschränkung der viralen Abwehr ein Abbruch erwogen werden. Nichtinterventionelle Beobachtungsstudien führen definitionsgemäß nicht zu vermehrtem Kontakt des Patienten mit dem Studienzentrum. Sollte ein durch SARS-CoV-2 veränderter Verlaufsbeobachtungsmodus erfolgen, sollte dies in der Beobachtungsstudie dokumentiert werden.

Empfehlungsgrad: EL5/Zustimmungsgrad: Konsens (93\%)

Interventionelle Studien, vor allem Zulassungsstudien stellen, für viele Patienten die einzige verbliebene Behandlungsmöglichkeit dar [126]. Das Ziel der steroidfreien Remission sollte auch in der SARS-CoV-2-Pandemie nicht verlassen werden. Insbesondere die für einige Patienten ansonsten anstehende langfristige und in Zeiten der SARS-CoV-2-Pandemie besonders risikoreiche Steroidtherapie [44] sollte unbedingt vermieden werden und es sollte explizit auch in der Pandemiezeit ein Studieneinschluss erwogen werden.

Der Wirkmechanismus des angewandten Präparats sollte auf Einschränkung der viralen Abwehr geprüft werden und ggfs. eine alternative Therapiemöglichkeit erwogen werden. Weiterhin kann der Wirkmechanismus des zu prüfenden Präparats zu einer Präferenz einer Studie gegenüber konkurrierenden Studien führen. Selbstverständlich sollten Studien unter den üblichen Schutzmaßnahmen durchgeführt werden.

Studien, in die bereits Patienten eingeschlossen worden sind, sollten nicht abgebrochen werden. Die Gründe sind die gleichen wie bei dem Einschluss in eine Studie. Bei Risikopatienten und Patienten in Studien mit einer Prüfsubstanz, die die virale Abwehr stark beeinträchtigt, sollte individuell das Fortsetzen der Studie abgewogen werden.

Nichtinterventionelle Beobachtungsstudien führen definitionsgemäß nicht zu vermehrtem Kontakt des Patienten mit dem Studienzentrum und können daher weiterhin durchgeführt werden. Sollte ein durch SARS-CoV-2 veränderter Verlaufsbeobachtungsmodus erfolgen, soll dieser in der Beobachtungsstudie dokumentiert und der Studienleitung mitgeteilt werden. Sollte der veränderte Verlaufsbeobachtungsmodus das primäre Studienziel beeinflussen, z. B. durch während der SARS-CoV-2-Pandemie erfolgte Telemedizin-Visiten, bei denen keine körperliche Untersuchung in das Studienergebnis einfließt, muss dies von der Studienleitung berücksichtigt werden. Maßnahmen wie Protokolländerungen, neue Fallzahlkalkulation oder Rekrutierungspause sollten dann von der Studienleitung erwogen werden.

\section{Thromboembolische Ereignisse}

\section{EMPFEHLUNG 4.3}

Hospitalisierte Patienten mit einer CED und COVID-19-Erkrankung sollten mindestens eine Thromboseprophylaxe erhalten. Bei ambulanten, an COVID-19 erkrankten CED-Patienten sollte entsprechend ihrem individuellen Risikoprofil und ihrer Begleitmedikation die Entscheidung für eine Thromboseprophylaxe großzügig getroffen werden.

Empfehlungsgrad: EL 3/Zustimmungsgrad: starker Konsens (95\%)

CED-Patienten haben im akuten Schub ein erhöhtes Risiko für thromboembolische Komplikationen [127]. Sie sollten daher entsprechend u. a. den gültigen DGVS-Leitlinien eine Thromboseprophylaxe erhalten $[4,128,129]$.

Bei Patienten mit schwerer COVID-19 wurde gehäuft über eine Hyperkoagulopathie mit Nachweis peripherer und zentraler Lungenembolien berichtet. Auch der Nachweis von Antiphospholipid-Antikörpern und multiple Infarkte wurden beschrieben. Dabei finden sich erhöhte D-Dimer-Spiegel, deren Anstieg im Krankheitsverlauf mit einer ungünstigen Krankheitsprognose verbunden ist [130]. Während eines schweren COVID-19-Verlaufs finden sich häufig auch eine aPTT-Verlängerung, ein vermehrter Anfall von Fibrinspaltprodukten und eine schwere Thrombozytopenie, die zu einer lebensbedrohlichen disseminierten intravasalen Koagulopathie (DIC) führen kann [130, 131].

Daher sollte die Indikation zur Einleitung einer pharmakologischen Thromboseprophylaxe mit niedermolekularem Heparin bei stationären COVID-19-Patienten (nicht nur bei CED) umgehend und bei ambulanten CED-Patienten großzügig entsprechend ihrem individuellen Risikoprofil und der Begleitmedikation gestellt werden [132]. Zu welchem Zeitpunkt bei einem schwer an COVID-19 erkrankten Patienten eine therapeutische Antikoagulation indiziert ist, muss im Rahmen des gesamten intensivmedizinischen Zusammenhangs entschieden werden und ist wahrscheinlich unabhängig von einer bestehenden CED.

\section{Ernährung}

\section{EMPFEHLUNG 4.4}

Der Ernährungsstatus von CED-Patienten sollte regelmäßig überwacht und Mangelzustände sollten ausgeglichen werden. Empfehlungsgrad: EL 5/Zustimmungsgrad: starker Konsens (96\%)

Eine Mangelernährung verstärkt eine medikamentös und krankheitsbedingt induzierte Immundefizienz [133]. Eine Mangelernährung ist ein unabhängiger Risikofaktor für das Auftreten von Komplikationen der CED und ungünstiger postoperativer Krankheitsverläufe [134, 135]. Daher sind die Prävention und die Behandlung der Malnutrition obligatorische Bestandteile der Therapie chronisch entzündlicher Darmerkrankungen (DGVS Leitlinie). 
Patienten mit aktiver CED haben im Vergleich zu gesunden Kontrollen eine höhere Prävalenz von Unterernährung. Die Leitlinien der DGVS zur Therapie des M. Crohn und der Colitis ulcerosa empfehlen daher, dass ein weiteres Screening stattfindet, wenn klinische Zeichen oder ein erhöhtes Risiko für eine Unterernährung oder einen Mangel an Nährstoffen vorliegen. Entsprechend des Ernährungszustands oder des Mangels an Mikronährstoffen soll dann ein Ausgleich stattfinden [136].

Auch wenn die Datenlage unbefriedigend ist, konnten die bisher durchgeführten Untersuchungen zeigen, dass der Ernährungsstatus des Wirts einen unabhängigen Risikofaktor für den Erkrankungsverlauf darstellt [137]. Diese aus der SARS- und der HIV-Infektion bekannten Daten zeigen, dass Patienten mit einem unzureichenden $\mathrm{Er}$ nährungszustand eine schlechtere Prognose haben. Wenn auch bisher zu diesem Thema bezüglich COVID-19 und Mangelernährung noch keine eindeutigen Daten vorliegen $[138,139]$, ist davon auszugehen, dass auch bei dieser Erkrankung ein unzureichender Ernährungszustand oder ein Mangel an Mikronährstoffen mit einem ungünstigeren Krankheitsverlauf verbunden sind.

\section{Interessenkonflikt}

Siehe Anhang

Literatur

[1] Ghebreyesus TA. WHO Director-General's opening remarks at the media briefing on COVID-19 - 11 March 2020. In. 2020 https://www.who.int/ $\mathrm{dg} /$ speeches/detail/who-director-general-s-opening-remarks-at-themedia-briefing-on-covid-19-11-march-2020

[2] Burisch J, Kiudelis G, Kupcinskas L et al. Natural disease course of Crohn's disease during the first 5 years after diagnosis in a European populationbased inception cohort: an Epi-IBD study. Gut 2018. doi:10.1136/ gutjnl-2017-315568

[3] Burisch J, Katsanos KH, Christodoulou DK et al. Natural Disease Course of Ulcerative Colitis During the First Five Years of Follow-up in a European Population-based Inception Cohort-An Epi-IBD Study. J Crohns Colitis 2019; 13: 198-208. doi:10.1093/ecco-jcc/jjy154

[4] Kucharzik T, Dignass AU, Atreya R et al. Updated S3-Guideline Ulcerative Colitis. German Society for Digestive and Metabolic Diseases (DGVS). Z Gastroenterol 2019; 57: 162-241

[5] Evidence OCfE-bMLo. 2020 http://www.cebm.net/index.aspx?o=1025, letzter Aufruf: 26.04.2020.

[6] Novel Coronavirus Pneumonia Emergency Response Epidemiology T. [The epidemiological characteristics of an outbreak of 2019 novel coronavirus diseases (COVID-19) in China]. Zhonghua Liu Xing Bing Xue Za Zhi 2020; 41: 145-151. doi:10.3760/cma.j.issn.0254-6450.2020.02.003

[7] Deng SQ, Peng HJ. Characteristics of and Public Health Responses to the Coronavirus Disease 2019 Outbreak in China. J Clin Med 2020; 9: doi: $10.3390 / \mathrm{jcm} 9020575$

[8] RKI. 2020 https://www.rki.de/DE/Content/InfAZ/N/Neuartiges_ Coronavirus/Fallzahlen.html letzter Aufruf am 16.05.2020.

[9] WHO. Report of the WHO-China Joint Mission on Coronavirus Disease 2019 (COVID-19). In. 2020 https://www.who.int/docs/default-source/ coronaviruse/who-china-joint-mission-on-covid-19-final-report.pdf

[10] Jin X, Lian JS, Hu JH et al. Epidemiological, clinical and virological characteristics of 74 cases of coronavirus-infected disease 2019 (COVID-19) with gastrointestinal symptoms. Gut 2020. doi:10.1136/gutjnl-2020320926
[11] Rodriguez-Lago I, Ramirez de la Piscina P, Elorza A et al. Characteristics and prognosis of patients with inflammatory bowel disease during the SARS-CoV-2 pandemic in the Basque Country (Spain). Gastroenterology 2020. doi:10.1053/j.gastro.2020.04.043

[12] IOIBD. 2020 https://covidibd.org/; letzter Aufruf am 12.05.2020

[13] Carvalho A, Alqusairi R, Adams A et al. SARS-CoV-2 Gastrointestinal Infection Causing Hemorrhagic Colitis: Implications for Detection and Transmission of COVID-19 Disease. The American journal of gastroenterology 2020. doi:10.14309/ajg.0000000000000667

[14] Chen L, Lou J, Bai Y et al. COVID-19 Disease With Positive Fecal and Negative Pharyngeal and Sputum Viral Tests. The American journal of gastroenterology 2020; 115: 790. doi:10.14309/ajg.0000000000000610

[15] Gu J, Han B, Wang J. COVID-19: Gastrointestinal Manifestations and Potential Fecal-Oral Transmission. Gastroenterology 2020; 158: 15181519. doi:10.1053/j.gastro.2020.02.054

[16] Chen N, Zhou M, Dong X et al. Epidemiological and clinical characteristics of 99 cases of 2019 novel coronavirus pneumonia in Wuhan, China: a descriptive study. Lancet 2020; 395: 507-513. doi:10.1016/ S0140-6736(20)30211-7

[17] Guan WJ, Ni ZY, Hu Y et al. Clinical Characteristics of Coronavirus Disease 2019 in China. N Engl J Med 2020. doi:10.1056/NEJMoa2002032

[18] Wu J, Liu J, Zhao X et al. Clinical Characteristics of Imported Cases of COVID-19 in Jiangsu Province: A Multicenter Descriptive Study. Clin Infect Dis 2020. doi:10.1093/cid/ciaa199

[19] Dreher M, Kersten A, Bickenbach J et al. Charakteristik von 50 hospitalisierten COVID-19-Patienten mit und ohne ARDS. Dtsch Arztebl Int 2020; 117: 271-278. doi:10.3238/arztebl.2020.0271

[20] ECDC. Coronavirus disease 2019 (COVID-19) in the EU/EEA and the UK- ninth update. 2020

[21] RKI. 2020 https://www.rki.de/DE/Content/InfAZ/N/Neuartiges_ Coronavirus/Steckbrief.html letzter Aufruf am 15.05.2020

[22] Grunert PC, Reuken PA, Stallhofer J et al. IBD in the COVID-19 pandemic - the patients' perspective. 2020

[23] Mao R, Liang J, Shen J et al. Implications of COVID-19 for patients with pre-existing digestive diseases. Lancet Gastroenterol Hepatol 2020; 5: 426-428. doi:10.1016/S2468-1253(20)30076-5

[24] Norsa L, Indriolo A, Sansotta N et al. Uneventful course in IBD patients during SARS-CoV-2 outbreak in northern Italy. Gastroenterology 2020. doi:10.1053/j.gastro.2020.03.062

[25] Taxonera C, Sagastagoitia I, Alba C et al. 2019 Novel Coronavirus Disease (COVID-19) in patients with Inflammatory Bowel Diseases. Alimentary pharmacology \& therapeutics 2020. doi:10.1111/apt.15804

[26] Allocca M, Fiorino G, Zallot C et al. Incidence and patterns of COVID-19 among inflammatory bowel disease patients from the Nancy and Milan cohorts. Clinical gastroenterology and hepatology: the official clinical practice journal of the American Gastroenterological Association; 2020. doi:10.1016/j.cgh.2020.04.071

[27] Lavezzo E, Franchin E, Ciavarella C et al. Suppression of COVID-19 outbreak in the municipality of Vo, Italy. medRxiv 2020. doi:10.1101/ 2020.04.17.20053157

[28] Gudbjartsson DF, Helgason A, Jonsson $\mathrm{H}$ et al. Spread of SARS-CoV-2 in the Icelandic Population. N Engl J Med 2020. doi:10.1056/ NEJMoa2006100

[29] Armann JPDN, Simon A, Doenhardt M et al. Hospital admission in children and adolescents with COVID-19 - early results from a national survey conducted by the German Society for Pediatric Infectious Diseases (DGPI). Dtsch Arztebl Int 2020; 117: 373-374. doi:10.3238/ arztebl.2020.0373

[30] Turner D, Huang Y, Martin-de-Carpi J et al. COVID-19 and Paediatric Inflammatory Bowel Diseases: Global Experience and Provisional Guidance (March 2020) from the Paediatric IBD Porto group of ESPGHAN. J Pediatr Gastroenterol Nutr 2020. doi:10.1097/mpg.0000000000002729 
[31] Tinsley A, Navabi S, Williams ED et al. Increased Risk of Influenza and Influenza-Related Complications Among 140,480 Patients With Inflammatory Bowel Disease. Inflamm Bowel Dis 2018. doi:10.1093/ibd/izy243

[32] Ko MK, Ng SC, Mak LY et al. Infection-related hospitalizations in the first year after inflammatory bowel disease diagnosis. Journal of digestive diseases 2016; 17: 610-617. doi:10.1111/1751-2980.12397

[33] Magro FRJ-F, Abeu C, McMahon E et al. Inflammatory bowel disease management during the COVID-19 outbreak: The 10 do's and don'ts from the ECCO-COVID Taskforce. J Crohns Colitis 2020

[34] Pin An M], Haixia R, Juan S et al. Protection of 318 Inflammatory Bowel Disease Patients from the Outbreak and Rapid Spread of COVID-19 Infection in Wuhan, China. The Lancet 2020; ePub ahead of print doi:10.2139/ssrn.3543590

[35] Bin Abdulrahman AK, Bin Abdulrahman KA, Almadi MK et al. Do various personal hygiene habits protect us against influenza-like illness? BMC Public Health 2019; 19: 1324. doi:10.1186/s12889-019-7726-9

[36] Cheng HY, Jian SW, Liu DP et al. Contact Tracing Assessment of COVID19 Transmission Dynamics in Taiwan and Risk at Different Exposure Periods Before and After Symptom Onset. JAMA internal medicine 2020. doi:10.1001/jamainternmed.2020.2020

[37] Wölfel R, Corman VM, Guggemos W et al. Virological assessment of hospitalized patients with COVID-2019. Nature 2020. doi:10.1038/ s41586-020-2196-x

[38] Ng K, Poon BH, Kiat Puar TH et al. COVID-19 and the Risk to Health Care Workers: A Case Report. Ann Intern Med 2020. doi:10.7326/L20-0175

[39] Zabana Y, Rodriguez L, Lobaton T et al. Relevant Infections in Inflammatory Bowel Disease, and Their Relationship With Immunosuppressive Therapy and Their Effects on Disease Mortality. Journal of Crohn's \& colitis 2019; 13: 828-837. doi:10.1093/ecco-jcc/jjz013

[40] Damiani G, Pacifico A, Bragazzi NL et al. Biologics increase the risk of SARS-CoV-2 infection and hospitalization, but not ICU admission and death: Real-life data from a large cohort during red-zone declaration. Dermatol Ther 2020: e13475. doi:10.1111/dth.13475

[41] Long MD, Martin C, Sandler RS et al. Increased risk of pneumonia among patients with inflammatory bowel disease. The American journal of gastroenterology 2013; 108: 240-248. doi:10.1038/ajg.2012.406

[42] Orlicka K, Barnes E, Culver EL. Prevention of infection caused by immunosuppressive drugs in gastroenterology. Therapeutic advances in chronic disease 2013; 4: 167-185. doi:10.1177/2040622313485275

[43] Dorrington AM, Selinger CP, Parkes GC et al. The historical role and contemporary use of corticosteroids in inflammatory bowel disease. J Crohns Colitis 2020. doi:10.1093/ecco-jcc/jjaa053

[44] Lichtenstein GR, Feagan BG, Cohen RD et al. Serious infection and mortality in patients with Crohn's disease: more than 5 years of follow-up in the TREAT registry. The American journal of gastroenterology 2012; 107: 1409-1422. doi:10.1038/ajg.2012.218

[45] Wisniewski AKJ, Seksik P, Landman C et al. the Saint-Antoine IBD network, Collaborators Saint Antoine IBD Network. Increased incidence of systemic serious viral infections in patients with inflammatory bowel disease associates with active disease and use of thiopurine. United European Gastroenterology Journal 2020; 8 (3): 303-313

[46] Seksik P, Cosnes J, Sokol $\mathrm{H}$ et al. Incidence of benign upper respiratory tract infections, HSV and HPV cutaneous infections in inflammatory bowel disease patients treated with azathioprine. Alimentary pharmacology \& therapeutics 2009; 29: 1106-1113. doi:10.1111/ j.1365-2036.2009.03973.x

[47] Kirchgesner J, Lemaitre M, Carrat F et al. Risk of Serious and Opportunistic Infections Associated With Treatment of Inflammatory Bowel Diseases. Gastroenterology 2018; 155: 337-346.e310. doi:10.1053/ j.gastro.2018.04.012
[48] Kochar B, Cai W, Cagan A et al. Pre-treatment Frailty Is Independently Associated With Increased Risk of Infections After Immunosuppression in Patients with Inflammatory Bowel Diseases. Gastroenterology 2020. doi:10.1053/j.gastro.2020.02.032

[49] Fleming SB. Viral Inhibition of the IFN-Induced JAK/STAT Signalling Pathway: Development of Live Attenuated Vaccines by Mutation of ViralEncoded IFN-Antagonists. Vaccines (Basel) 2016; 4: doi:10.3390/ vaccines 4030023

[50] Kelesidis T, Mastoris I, Metsini A et al. How to approach and treat viral infections in ICU patients. BMC Infect Dis 2014; 14: 321. doi:10.1186/ 1471-2334-14-321

[51] Favalli EG, Biggioggero M, Maioli G et al. Baricitinib for COVID-19: a suitable treatment? The Lancet Infectious diseases 2020 doi:10.1016/S1473-3099(20)30262-0

[52] Feagan BG, Bhayat F, Khalid M et al. Respiratory Tract Infections in Patients With Inflammatory Bowel Disease: Safety Analyses From Vedolizumab Clinical Trials. J Crohns Colitis 2018; 12: 905-919. doi:10.1093/ecco-jcc/jjy047

[53] Zabotti A, Goletti D, Lubrano E et al. The impact of the interleukin 12/23 inhibitor ustekinumab on the risk of infections in patients with psoriatic arthritis. Expert Opin Drug Saf 2020; 19: 69-82. doi:10.1080/ 14740338.2020.1703946

[54] Rubin DT, Abreu MT, Rai V et al. Management of Patients with Crohn's Disease and Ulcerative Colitis During the COVID-19 Pandemic: Results of an International Meeting. Gastroenterology 2020. doi:10.1053/ j.gastro.2020.04.002

[55] Linton NM, Kobayashi T, Yang Y et al. Incubation Period and Other Epidemiological Characteristics of 2019 Novel Coronavirus Infections with Right Truncation: A Statistical Analysis of Publicly Available Case Data. J Clin Med 2020; 9: doi:10.3390/jcm9020538

[56] Toruner M, Loftus EV Jr, Harmsen WS et al. Risk factors for opportunistic infections in patients with inflammatory bowel disease. Gastroenterology 2008; 134: 929-936

[57] Tosca J, Garcia N, Pascual I et al. Clinical assessment of risk factors for infection in inflammatory bowel disease patients. Int J Colorectal Dis 2020; 35: 491-500. doi:10.1007/s00384-019-03501-0

[58] Bezzio C, Saibeni S, Variola A et al. Outcomes of COVID-19 in 79 patients with IBD in Italy: an IG-IBD study. Gut 2020. doi:10.1136/gutjnl-2020321411

[59] (ISS) ISdS. Sorveglianza Integrata COVID-19 in Italia 2020. 2020 https://wwwepicentroissit/coronavirus/bollettino/Infografica_ 26marzo\%20ITApdf)

[60] RKI. Täglicher Lagebericht des RKI zur Coronavirus-Krankheit-2019 (COVID-19). 27.04-AKTUALISIERTER STAND FÜR DEUTSCHLAND. 2020 https://wwwrkide/DE/Content/InfAZ/N/Neuartiges_Coronavirus/ Situationsberichte/2020-04-27-depdf?_blob=publicationFile

[61] Statista. 2020 https://de.statista.com/statistik/daten/studie/1365/ umfrage/bevoelkerung-deutschlands-nach-altersgruppen/ letzter Aufruf am 01.05.2020

[62] Destatis. 2020 https://www.destatis.de/DE/Presse/Pressemitteilungen/ 2020/03/PD20_115_132.html;jsessionid=7F326250BEDCBE087ABE 219A28DE59F5.internet8712; letzter Aufruf am 01.05.2020

[63] Soziales BfAu. 2020 https://www.bmas.de/DE/Presse/Pressemitteilungen/ 2020/einheitlicher-arbeitsschutz-gegen-coronavirus.html; letzter Aufruf am 01.05.2020

[64] Colombel JF, D’Haens G, Lee WJ et al. Outcomes and Strategies to Support a Treat-to-target Approach in Inflammatory Bowel Disease: A Systematic Review. J Crohns Colitis 2020; 14: 254-266. doi:10.1093/ecco-jcc/jjz131

[65] Strohl M, Gonczi L, Kurt Z et al. Quality of care in inflammatory bowel diseases: What is the best way to better outcomes? World journal of gastroenterology 2018; 24: 2363-2372. doi:10.3748/wjg.v24.i22.2363 
[66] Huang C, Wang Y, Li X et al. Clinical features of patients infected with 2019 novel coronavirus in Wuhan, China. Lancet 2020; 395: 497-506. doi:10.1016/50140-6736(20)30183-5

[67] Occhipinti V, Pastorelli L. Challenges in the Care of IBD Patients During the CoViD-19 Pandemic: Report From a "Red Zone" Area in Northern Italy. Inflamm Bowel Dis 2020. doi:10.1093/ibd/izaa084

[68] de Jong MJ, Boonen A, van der Meulen-de Jong AE et al. Cost-effectiveness of Telemedicine-directed Specialized vs Standard Care for Patients With Inflammatory Bowel Diseases in a Randomized Trial. Clin Gastroenterol Hepatol 2020. doi:10.1016/j.cgh.2020.04.038

[69] de Jong MJ, van der Meulen-de Jong AE, Romberg-Camps MJ et al. Telemedicine for management of inflammatory bowel disease (mylBDcoach): a pragmatic, multicentre, randomised controlled trial. Lancet 2017; 390: 959-968. doi:10.1016/S0140-6736(17)31327-2

[70] RKI. 2020 https://www.rki.de/DE/Content/InfAZ/N/Neuartiges_ Coronavirus/nCoV.html; letzter Aufruf am 08.05.2020

[71] RKI. 2020 https://www.kvn.de/Information+zum+Coronavirus+\% 28SARS_CoV2+COVID+19\%29-p-6072.html; letzter Aufruf am 01.05 .2020

[72] Heida A, Park KT, van Rheenen PF. Clinical Utility of Fecal Calprotectin Monitoring in Asymptomatic Patients with Inflammatory Bowel Disease: A Systematic Review and Practical Guide. Inflamm Bowel Dis 2017; 23: 894-902. doi:10.1097/MIB.0000000000001082

[73] Sipponen T, Kolho KL. Fecal calprotectin in diagnosis and clinical assessment of inflammatory bowel disease. Scand J Gastroenterol 2015; 50: 74-80. doi:10.3109/00365521.2014.987809

[74] Haisma SM, Galaurchi A, Almahwzi S et al. Head-to-head comparison of three stool calprotectin tests for home use. PLoS One 2019; 14: e0214751. doi:10.1371/journal.pone.0214751

[75] Kucharzik T, Wittig BM, Helwig U et al. Use of Intestinal Ultrasound to Monitor Crohn's Disease Activity. Clinical gastroenterology and hepatology: the official clinical practice journal of the American Gastroenterological Association 2017; 15: 535-542 e532. doi:10.1016/ j.cgh.2016.10.040

[76] Maaser C, Petersen F, Helwig U et al. Intestinal ultrasound for monitoring therapeutic response in patients with ulcerative colitis: results from the TRUST\&UC study. Gut 2019. doi:10.1136/gutjnl-2019-319451

[77] Repici A, Pace F, Gabbiadini R et al. Endoscopy units and the COVID-19 Outbreak: A Multi-Center Experience from Italy. Gastroenterology 2020. doi:10.1053/j.gastro.2020.04.003

[78] Repici A, Maselli R, Colombo M et al. Coronavirus (COVID-19) outbreak: what the department of endoscopy should know. Gastrointest Endosc 2020. doi:10.1016/j.gie.2020.03.019

[79] Johnston ER, Habib-Bein N, Dueker JM et al. Risk of bacterial exposure to the endoscopist's face during endoscopy. Gastrointest Endosc 2019; 89: 818-824. doi:10.1016/j.gie.2018.10.034

[80] van Doremalen N, Bushmaker T, Morris DH et al. Aerosol and Surface Stability of SARS-CoV-2 as Compared with SARS-CoV-1. N Engl J Med 2020; 382: 1564-1567. doi:10.1056/NEJMc2004973

[81] Gu J, Han B, Wang J. COVID-19: Gastrointestinal Manifestations and Potential Fecal-Oral Transmission. Gastroenterology 2020 doi:10.1053/j.gastro.2020.02.054

[82] Xiao F, Tang M, Zheng X et al. Evidence for Gastrointestinal Infection of SARS-CoV-2. Gastroenterology 2020. doi:10.1053/j.gastro.2020.02.055

[83] Song Y, Liu P, Shi XL et al. SARS-CoV-2 induced diarrhoea as onset symptom in patient with COVID-19. Gut 2020. doi:10.1136/ gutjnl-2020-320891

[84] Hamming I, Timens W, Bulthuis ML et al. Tissue distribution of ACE2 protein, the functional receptor for SARS coronavirus. A first step in understanding SARS pathogenesis. J Pathol 2004; 203: 631-637. doi:10.1002/path. 1570
[85] Prevention. CfDCa. Coronavirus Disease 2019 (COVID-19). Available at (letzter Aufruf am 15.05.2020): 2020 https://www.cdc.gov/coronavirus/ 2019-ncov/infection-control/infection-prevention-control-faq.html

[86] Control. ECfDPa. Infection prevention and control for COVID-19 in healthcare settings. Available at (letzter Aufruf am 15.05.2020): 2020 https://www.ecdc.europa.eu/en/publications-data/infectionprevention-and-control-covid-19-healthcare-settings

[87] Gralnek IM, Hassan C, Beilenhoff U et al. ESGE and ESGENA Position Statement on gastrointestinal endoscopy and the COVID-19 pandemic. Endoscopy 2020. doi:10.1055/a-1155-6229

[88] Sultan S, Lim JK, Altayar O et al. AGA Institute Rapid Recommendations for Gastrointestinal Procedures During the COVID-19 Pandemic. Gastroenterology 2020. doi:10.1053/j.gastro.2020.03.072

[89] Chiu PWY, Ng SC, Inoue H et al. Practice of endoscopy during COVID-19 pandemic: position statements of the Asian Pacific Society for Digestive Endoscopy (APSDE-COVID statements). Gut 2020. doi:10.1136/ gutjnl-2020-321185

[90] Han J, Wang Y, Zhu L et al. Preventing the spread of COVID-19 in digestive endoscopy during the resuming period: meticulous execution of screening procedures. Gastrointest Endosc 2020. doi:10.1016/ j.gie.2020.03.3855

[91] lacucci M, Cannatelli R, Labarile $\mathrm{N}$ et al. Endoscopy in inflammatory bowel diseases during the COVID-19 pandemic and post-pandemic period. Lancet Gastroenterol Hepatol 2020. doi:10.1016/\$24681253(20)30119-9

[92] Neumann H, Emura F, Bokemeyer B et al. Practical Advice for Management of IBD Patients during the COVID-19 Pandemic: A World Endoscopy Organization Statement. Dig Endosc 2020. doi:10.1111/ den. 13712

[93] Vavricka SR, Tutuian R, Imhof A et al. Air suctioning during colon biopsy forceps removal reduces bacterial air contamination in the endoscopy suite. Endoscopy 2010; 42: 736-741. doi:10.1055/s-0030-1255615

[94] Neurath MF. Covid-19 and immunomodulation in IBD. Gut 2020. doi:10.1136/gutjnl-2020-321269

[95] Euroimmun. 2020 https://www.coronavirus-diagnostik.de; letzter Aufruf am 01.05.2020

[96] Euroimmun. https://www.coronavirus-diagnostik.de/documents/ Indications/Infections/Coronavirus/YI_2606_I_DE_B.pdf. 2020. letzter Aufruf am 01.05.2020

[97] Streeck Hea. Vorläufiges Ergebnis und Schlussfolgerungen der COVID-19 Case Cluster Study (Gemeinde Gangelt) in land. nrw 942020 2020: S4

[98] Taskforce. PDCAobotC-E. 2020 https://ecco-ibd.eu/images/6_Publication/ 6_8_Surveys/1st_interview_COVID-19\%20ECCOTaskforce_published.pdf; letzter Aufruf am 29.03.2020

[99] Papamichael K, Karatzas P, Mantzaris G]. De-escalation of Infliximab Maintenance Therapy from 8- to 10-week Dosing Interval Based on Faecal Calprotectin in Patients with Crohn's Disease. J Crohns Colitis 2016; 10: 371-372. doi:10.1093/ecco-jcc/jjv206

[100] Giwa AL, Desai A, Duca A. Novel 2019 coronavirus SARS-CoV-2 (COVID-19): An updated overview for emergency clinicians. Emergency medicine practice 2020; 22: 1-28

[101] Russell CD, Millar JE, Baillie JK. Clinical evidence does not support corticosteroid treatment for 2019-nCoV lung injury. Lancet 2020; 395: 473-475. doi:10.1016/s0140-6736(20)30317-2

[102] Wu C, Chen X, Cai Y et al. Risk Factors Associated With Acute Respiratory Distress Syndrome and Death in Patients With Coronavirus Disease 2019 Pneumonia in Wuhan, China. JAMA internal medicine 2020. doi:10.1001/jamainternmed.2020.0994

[103] Ling Y, Xu SB, Lin YX et al. Persistence and clearance of viral RNA in 2019 novel coronavirus disease rehabilitation patients. Chinese medical journal 2020; 133: 1039-1043. doi:10.1097/cm9.0000000000000774 
[104] Liang-Ru Zhu RM, Gionata Fiorino, Thomas Schneider on behalf of the COVID-19 ECCO Taskforce. 2020 https://ecco-ibd.eu/images/ 6_Publication/6_8_Surveys/2nd_Interview_COVID-19_ECCO_ Taskforce_published.pdf; letzter Aufruf am 29.03.2020

[105] Wu D, Yang XO. TH17 responses in cytokine storm of COVID-19: An emerging target of JAK2 inhibitor Fedratinib. J Microbiol Immunol Infect 2020. doi:10.1016/j.jmii.2020.03.005

[106] Winthrop KL, Melmed GY, Vermeire S et al. Herpes Zoster Infection in Patients With Ulcerative Colitis Receiving Tofacitinib. Inflamm Bowel Dis 2018; 24: 2258-2265. doi:10.1093/ibd/izy131

[107] Boor PPC, de Ruiter PE, Asmawidjaja PS et al. JAK-inhibitor tofacitinib suppresses interferon alfa production by plasmacytoid dendritic cells and inhibits arthrogenic and antiviral effects of interferon alfa. Transl Res 2017; 188: 67-79. doi:10.1016/j.trsl.2016.11.006

[108] Jamilloux Y, Henry T, Belot A et al. Should we stimulate or suppress immune responses in COVID-19? Cytokine and anti-cytokine interventions. Autoimmunity reviews 2020. doi:10.1016/j.autrev.2020.102567

[109] Stebbing J, Phelan A, Griffin I et al. COVID-19: combining antiviral and anti-inflammatory treatments. The Lancet Infectious diseases 2020; 20: 400-402. doi:10.1016/s1473-3099(20)30132-8

[110] Spinelli FR, Conti F, Gadina M. HiJAKing SARS-CoV-2? The potential role of JAK inhibitors in the management of COVID-19. Science immunology 2020; 5 . doi: $10.1126 /$ sciimmunol.abc5367

[111] Torres J, Bonovas S, Doherty G et al. ECCO Guidelines on Therapeutics in Crohn's Disease: Medical Treatment. J Crohns Colitis 2020; 14: 4-22. doi:10.1093/ecco-jcc/jjz180

[112] Roblin X, Boschetti G, Williet N et al. Azathioprine dose reduction in inflammatory bowel disease patients on combination therapy: an open-label, prospective and randomised clinical trial. Alimentary pharmacology \& therapeutics 2017; 46: 142-149. doi:10.1111/apt.14106

[113] Boyapati RK, Torres J, Palmela C et al. Withdrawal of immunosuppressant or biologic therapy for patients with quiescent Crohn's disease. The Cochrane database of systematic reviews 2018; 5: Cd012540. doi:10.1002/14651858.CD012540.pub2

[114] Van Assche G, Magdelaine-Beuzelin C, D’Haens G et al. Withdrawal of immunosuppression in Crohn's disease treated with scheduled infliximab maintenance: a randomized trial. Gastroenterology 2008; 134: 1861-1868. doi:10.1053/j.gastro.2008.03.004

[115] Chen L, Liu HG, Liu W et al. [Analysis of clinical features of 29 patients with 2019 novel coronavirus pneumonia]. Zhonghua Jie He He Hu Xi Za Zhi 2020; 43: 203-208. doi:10.3760/cma.j.issn.10010939.2020 .03 .013

[116] Mehta AK, Gracias DT, Croft M. TNF activity and T cells. Cytokine 2018; 101: 14-18. doi:10.1016/j.cyto.2016.08.003

[117] Ng SC, Hilmi IN, Blake A et al. Low Frequency of Opportunistic Infections in Patients Receiving Vedolizumab in Clinical Trials and PostMarketing Setting. Inflamm Bowel Dis 2018; 24: 2431-2441. doi:10.1093/ibd/izy153

[118] Van Assche G, Vermeire S, Noman M et al. Infliximab administered with shortened infusion times in a specialized IBD infusion unit: a prospective cohort study. J Crohns Colitis 2010; 4: 329-333. doi:10.1016/ j.crohns.2009.12.012

[119] Fukuyo S, Saito K, Yamaoka K et al. Efficacy and safety of reducing duration of infliximab infusion. Modern rheumatology 2014; 24: 275280. doi:10.3109/14397595.2013.843747

[120] El-Matary W, Dykes DMH, Bauman L et al. Rapid Infliximab Infusion in Children with Inflammatory Bowel Disease: A Multicenter North American Experience. Inflamm Bowel Dis 2017; 23: 2104-2108. doi:10.1097/mib.0000000000001259

[121] Sandborn W], Baert F, Danese S et al. Efficacy and Safety of Vedolizumab Subcutaneous Formulation in a Randomized Trial of Patients With Ulcerative Colitis. Gastroenterology 2020; 158: 562-572.e512. doi:10.1053/j.gastro.2019.08.027
[122] Ruemmele FM, Veres G, Kolho KL et al. Consensus guidelines of ECCO/ ESPGHAN on the medical management of pediatric Crohn's disease. J Crohns Colitis 2014; 8: 1179-1207. doi:10.1016/j.crohns.2014.04.005

[123] Stevens T, Haasnoot LDHG, Buskens $C$ et al. Reduced need for surgery and medical therapy after early ileocaecal resection for Crohn's disease: Long-term follow-up of the LIR!C trial. Journal of Crohn's and Colitis 2020; 14: S003-S004

[124] Ponsioen CY, de Groof EJ, Eshuis EJ et al. Laparoscopic ileocaecal resection versus infliximab for terminal ileitis in Crohn's disease: a randomised controlled, open-label, multicentre trial. The lancet Gastroenterology \& hepatology 2017; 2: 785-792. doi:10.1016/S24681253(17)30248-0

[125] RKI. Epidemiologisches Bulletin 18 | 2020 30. April 2020. letzter Aufruf am 01.05.2020 2020

[126] Sachar DB. Placebo-controlled clinical trials in gastroenterology. A position paper of the American College of Gastroenterology. The American journal of gastroenterology 1984; 79: 913-917

[127] Grainge M], West J, Card TR. Venous thromboembolism during active disease and remission in inflammatory bowel disease: a cohort study. Lancet 2010; 375: 657-663. doi:10.1016/s0140-6736(09)61963-2

[128] Preiß JC, Bokemeyer B, Buhr H] et al. [Updated German clinical practice guideline on “Diagnosis and treatment of Crohn's disease” 2014]. Z Gastroenterol 2014; 52: 1431-1484. doi:10.1055/s-0034-1385199

[129] Nguyen GC, Bernstein CN, Bitton A et al. Consensus statements on the risk, prevention, and treatment of venous thromboembolism in inflammatory bowel disease: Canadian Association of Gastroenterology. Gastroenterology 2014; 146: 835-848.e836. doi:10.1053| j.gastro.2014.01.042

[130] Terpos E, Ntanasis-Stathopoulos I, Elalamy I et al. Hematological findings and complications of COVID-19. American journal of hematology 2020. doi:10.1002/ajh.25829

[131] Llitjos JF, Leclerc M, Chochois C et al. High incidence of venous thromboembolic events in anticoagulated severe COVID-19 patients. Journal of thrombosis and haemostasis: JTH 2020. doi:10.1111/jth.14869

[132] Kollias A, Kyriakoulis KG, Dimakakos E et al. Thromboembolic risk and anticoagulant therapy in COVID-19 patients: emerging evidence and call for action. British journal of haematology 2020. doi:10.1111/ bjh.16727

[133] Balestrieri P, Ribolsi M, Guarino MPL et al. Nutritional Aspects in Inflammatory Bowel Diseases. Nutrients 2020; 12: doi:10.3390/ nu12020372

[134] Massironi S, Rossi RE, Cavalcoli FA et al. Nutritional deficiencies in inflammatory bowel disease: therapeutic approaches. Clinical nutrition (Edinburgh, Scotland) 2013; 32: 904-910. doi:10.1016/ j.clnu.2013.03.020

[135] Nguyen GC, Munsell M, Harris ML. Nationwide prevalence and prognostic significance of clinically diagnosable protein-calorie malnutrition in hospitalized inflammatory bowel disease patients. Inflamm Bowel Dis 2008; 14: 1105-1111. doi:10.1002/ibd.20429

[136] Schreiner P, Martinho-Grueber M, Studerus D et al. Nutrition in Inflammatory Bowel Disease. Digestion 2020. doi:10.1159/000505368

[137] Beck MA, Handy J, Levander OA. Host nutritional status: the neglected virulence factor. Trends in microbiology 2004; 12: 417-423. doi:10.1016/j.tim.2004.07.007

[138] Calder PC, Carr AC, Gombart AF et al. Optimal Nutritional Status for a Well-Functioning Immune System Is an Important Factor to Protect against Viral Infections. Nutrients 2020; 12: doi:10.3390/nu12041181

[139] Gasmi A, Noor S, Tippairote T et al. Individual risk management strategy and potential therapeutic options for the COVID-19 pandemic. Clinical immunology (Orlando, Fla) 2020. doi:10.1016/ j.clim.2020.108409 